\title{
Kubo Fluctuation Relations in the Generalized Elastic Model
}

\author{
Alessandro Taloni \\ Center for Complexity and Biosystems, Department of Physics, University of Milan, Via Celoria 16, 20133 Milan, Italy \\ Correspondence should be addressed to Alessandro Taloni; alessandro.taloni@gmail.com
}

Received 30 September 2015; Accepted 8 November 2015

Academic Editor: Soheil Salahshour

Copyright (C) 2016 Alessandro Taloni. This is an open access article distributed under the Creative Commons Attribution License, which permits unrestricted use, distribution, and reproduction in any medium, provided the original work is properly cited.

The generalized elastic model encompasses several linear stochastic models describing the dynamics of polymers, membranes, rough surfaces, and fluctuating interfaces. In this paper we show that the Fractional Langevin Equation (FLE) is a suitable framework for the study of the tracer (probe) particle dynamics, when an external force acts only on a single point $\vec{x}^{\star}$ (tagged probe) belonging to the system. With the help of the Fox function formalism we study the scaling behaviour of the noise- and force-propagators for large and short times (distances). We show that the Kubo fluctuation relations are exactly fulfilled when a time periodic force is exerted on the tagged probe. Most importantly, by studying the large and low frequency behaviour of the complex mobility we illustrate surprising nontrivial physical scenarios. Our analysis shows that the system splits into two distinct regions whose size depends on the applied frequency, characterized by very different response to the periodic perturbation exerted, both in the phase shift and in the amplitude.

\section{Introduction}

The generalized elastic model (GEM) has been firstly introduced in [1] by the following equation:

$$
\begin{aligned}
\frac{\partial}{\partial t} \mathbf{h}(\vec{x}, t)= & \int \Lambda\left(\vec{x}-\vec{x}^{\prime}\right) \frac{\partial^{z}}{\partial\left|\vec{x}^{\prime}\right|^{z}} \mathbf{h}\left(\vec{x}^{\prime}, t\right) d^{d} x^{\prime} \\
& +\boldsymbol{\eta}(\vec{x}, t) .
\end{aligned}
$$

The general formulation in (1) is given for the $D$-dimensional stochastic field $\mathbf{h}$ defined in the $d$-dimensional infinite space $\vec{x}$. The white noise satisfies the fluctuation-dissipation (FD) relation; that is,

$$
\left\langle\eta_{j}(\vec{x}, t) \eta_{k}\left(\vec{x}^{\prime}, t^{\prime}\right)\right\rangle=2 k_{B} T \Lambda\left(\vec{x}-\vec{x}^{\prime}\right) \delta_{j k} \delta\left(t-t^{\prime}\right)
$$

$(j, k \in[1, D])$, where $\Lambda(\vec{r})=1 /|\vec{r}|^{\alpha}$ corresponds to the hydrodynamic friction kernel whose Fourier transform is

$$
\Lambda(\vec{q})=\frac{(4 \pi)^{d / 2}}{2^{\alpha}} \frac{\Gamma((d-\alpha) / 2)}{\Gamma(\alpha / 2)}|\vec{q}|^{\alpha-d}=A|\vec{q}|^{\alpha-d}
$$

if $(d-1) / 2<\alpha<d$. The fractional derivative $\partial^{z} / \partial|\vec{x}|^{z}$, defined via its Fourier transform by [2]

$$
\mathscr{F}_{\vec{q}}\left\{\frac{\partial^{z}}{\partial|\vec{x}|^{z}}\right\} \equiv-|\vec{q}|^{z},
$$

has another common definition given in terms of the Laplacian $\Delta$ as $\partial^{z} / \partial|\vec{x}|^{z}:=-(-\Delta)^{z / 2}$ [3]. GEM (1) governs the dynamics of polymers [4-7], elastic chains [8-10], membranes [5, 11-15], and rough surfaces [16-21] among others. Equation (1) also reproduces the anomalous diffusive behaviour of systems such as crack propagation [22] and contact line of a liquid meniscus [23]. Each one of the abovementioned physical systems corresponds to a given set of the parameters defining GEM (1), namely, $z, \alpha$, and $d$ [24], with $\alpha=d$ in the case of $\Lambda(\vec{r})=\delta(|\vec{r}|)(A=$ const in (3)).

In $[24,25]$ we have considered the case of GEM (1) with an external local perturbation, namely, a force acting only on the probe in $\vec{x}^{\star}$, hereafter called the tagged probe. Under such condition, (1) transforms to the following stochastic evolution equation:

$$
\begin{aligned}
& \frac{\partial}{\partial t} \mathbf{h}(\vec{x}, t)=\int \Lambda\left(\vec{x}-\vec{x}^{\prime}\right)\left[\frac{\partial^{z}}{\partial\left|\vec{x}^{\prime}\right|^{z}} \mathbf{h}\left(\vec{x}^{\prime}, t\right)\right. \\
& \left.+\mathbf{F}\left\{\mathbf{h}\left(\vec{x}^{\prime}, t\right), t\right\} \delta\left(\vec{x}^{\prime}-\vec{x}^{\star}\right)\right] d^{d} x^{\prime} \\
& +\boldsymbol{\eta}(\vec{x}, t) .
\end{aligned}
$$


Here $\mathbf{F}\{\mathbf{h}(\vec{x}, t), t\}$ is a force functional of the stochastic fields $\mathbf{h}(\vec{x}, t)$ and of the time $t$ : it represents the external perturbation applied to the probe particle placed at the position $\vec{x}^{\star}$.

In this paper we show that the Kubo fluctuation relations rigorously hold when the applied perturbation is a time periodic force. To the best of my knowledge, the problem of the study of the response of a generalized elastic system to a time periodic perturbation has never been treated, not in the context of polymers or membranes nor when one is concerned with the dynamics of rough surfaces. The performed analysis demonstrates that the macroscopic effects are persistent in time. Indeed the system splits into two macroregions whose size is defined by the value of the applied frequency $\omega_{0}$ : the responses of these regions differ markedly in amplitude and phase, allowing us to make experimentally testable predictions about the viscoelastic properties of the system. We show this by adopting the Fox $H$-function formalism, which constitutes an excellent tool for the compact representation and the systematic analysis of the scaling properties of any correlation function and drift. Our analysis shows that the low and high frequency behaviours of the system response to the external perturbation reveal an extremely rich physical scenario in both the response amplitude and the phase. We also show that the $H$-functions describe properly the scaling of the noise- and force-propagators in the limit of large and short times (distances).

Fox $H$-functions are defined through the Mellin transform

$$
\begin{aligned}
& H_{p q}^{m n}\left[y \mid \begin{array}{ccc}
\left(a_{1}, A_{1}\right) & \cdots & \left(a_{p}, A_{p}\right) \\
\left(b_{1}, B_{1}\right) & \cdots & \left(b_{q}, B_{q}\right)
\end{array}\right] \\
& \quad=\frac{1}{2 \pi i} \int_{L} \chi(s) y^{-s} d s
\end{aligned}
$$

with $1 \leq m \leq q$ and $0 \leq n \leq p \cdot \chi(s)$ is given by

$$
\chi(s)=\frac{\prod_{j=1}^{m} \Gamma\left(b_{j}+B_{j} s\right) \prod_{j=1}^{n} \Gamma\left(1-a_{j}-A_{j} s\right)}{\prod_{j=m+1}^{q} \Gamma\left(1-b_{j}-B_{j} s\right) \prod_{j=n+1}^{p} \Gamma\left(a_{j}+A_{j} s\right)},
$$

where $A_{j}$ and $B_{j}$ are positive numbers, while $a_{j}$ and $b_{j}$ are complex. Empty products are interpreted as being unity. For convenience throughout this paper we adopt the following short notation:

$$
\begin{gathered}
H_{p q}^{m n}\left[y\left[\begin{array}{ccc}
\left(a_{1}, A_{1}\right) & \cdots & \left(a_{p}, A_{p}\right) \\
\left(b_{1}, B_{1}\right) & \cdots & \left(b_{q}, B_{q}\right)
\end{array}\right]\right. \\
=H_{p q}^{m n}\left[\begin{array}{c|c}
{\left[\begin{array}{l}
\left.a_{p}, A_{p}\right] \\
{\left[b_{q}, B_{q}\right]}
\end{array}\right] .}
\end{array}\right.
\end{gathered}
$$

In general, Fox $H$-functions gain more and more popularity among the scientific community, for their very general nature, which allows tackling different phenomena in a unified and elegant framework. Applications include nonDebye relaxation processes [26, 27], anomalous diffusion [28-31], reaction-diffusion equations [32], relaxation and reaction processes in disordered systems [33], and fractional Schrödinger equation [34], to name a few. The book [35] serves a deep analysis of properties of the Fox $H$-functions. Recent monograph [36] lists many useful properties of the Fox $H$-functions, together with some applications, for example, in astrophysics. The handbook [37] contains the list of useful properties and integrals of the Fox $H$-functions.

This paper is organized according to the following scheme. In Section 2 we recall the FLE framework in the absence of any applied force. We also report the two-point two-time correlation functions making use of the Fox $\mathrm{H}$ function formalism. In Section 2.2 we study the scaling short and long times behaviour of the noise-propagator in case of long range and local hydrodynamics. Section 3 is devoted to the FLE under the action of a localized potential; in particular in Section 3.1 we derive the scaling limits of the noisepropagator. In Section 4 we deal with the situation where the force applied to the probe particle in $\vec{x}^{\star}$ is time periodic: we study the scaling properties of the probe average velocity drifts, demonstrating the validity of the Kubo fluctuation relations (KFR) and Green-Kubo relation. We also derive exact results for the single file model and compare them with the outcomes of numerical simulations. In Appendix A we list the Fox $H$-functions properties that we use in our analysis; in Appendix B we report the technique for the asymptotic solution of Fourier integrals; in Appendix C Olver's theorem for solving integrals in the asymptotic limit, using the Laplace's method, is shown; in Appendix D we derive the exact expression for the Riemann-Liouville fractional derivative applied to an exponential function.

\section{Fractional Langevin Equation}

Equation (1) governs the dynamics of a linear stochastic system immersed in a viscous fluid. Let us take a different point of view: let us consider the dynamics of a tagged point (hereafter named probe or tracer without distinction) on the system described by (1). The probe can represent a spot on a membrane surface [13], or a tracer particle among those composing a single file system $[38,39]$, or a tagged monomer in polymeric chains [40]. In [1] it was shown that the stochastic equation describing the motion of the probe coordinate $h=h_{j}$ placed at position $\vec{x}$ is the following Fractional Langevin Equation:

$$
K_{-\infty}^{+} D_{t}^{\beta} h(\vec{x}, t)=\zeta(\vec{x}, t),
$$

where

$$
\begin{aligned}
\beta & =\frac{z-d}{z+\alpha-d}, \\
K^{+} & =\pi^{d / 2-1} \frac{\Gamma(d / 2) \sin (\pi \beta) \gamma}{2^{2-d} A^{\beta}}, \\
\gamma & =2(z+\alpha-d) .
\end{aligned}
$$


The pseudodifferential operator

$$
\begin{aligned}
{ }_{a} D_{t}^{\beta} \phi(t)=\frac{1}{\Gamma(1-\beta)} \frac{d}{d t} \int_{a}^{t} \frac{1}{\left(t-t^{\prime}\right)^{\beta}} \phi\left(t^{\prime}\right) d t^{\prime}, & \\
& 0<\beta<1,
\end{aligned}
$$

represents the left side Riemann-Liouville derivative with lower bound $a<t[3,41]$. We remind the reader that the derivative (13) is equivalent to the Caputo [42] derivative; that is,

$$
\frac{d^{\beta}}{d t^{\beta}} X(t)=\frac{1}{\Gamma(1-\beta)} \int_{a}^{t} \frac{\dot{X}\left(t^{\prime}\right)}{\left(t-t^{\prime}\right)^{\beta}} d t^{\prime}, \quad 0<\beta<1,
$$

if the lower bound in (13) is set as $a=-\infty$ [41].

The tracer dynamical representation provided by (9) is only valid whenever $z>d$; that is, $\beta<1((d-1) / 2<\alpha<$ $d)$. In the case of local hydrodynamics $(\Lambda(\vec{r})=\delta(|\vec{r}|))$ it is sufficient to set $\alpha=d$ and $A=$ const. The noise appearing on the RHS of (9) is defined as

$$
\begin{aligned}
& \zeta(\vec{x}, t) \\
& \quad=\int_{-\infty}^{+\infty} \int_{-\infty}^{t} \eta\left(\vec{x}^{\prime}, t^{\prime}\right) \Phi\left(\left|\vec{x}^{\prime}-\vec{x}\right|, t-t^{\prime}\right) d t^{\prime} d \vec{x}^{\prime},
\end{aligned}
$$

where we have introduced the noise-propagator $\Phi(|\vec{x}|, t)$ as $[24,25]$

$$
\begin{aligned}
& \Phi(|\vec{x}|, t)=\frac{K^{+}|\vec{x}|^{1-d / 2}}{(2 \pi)^{d / 2}} \\
& \cdot{ }_{0} D_{t}^{\beta} \int_{0}^{+\infty}|\vec{q}|^{d / 2} J_{d / 2-1}(|\vec{q}||\vec{x}|) e^{-A|\vec{q}|^{\gamma / 2} t} d|\vec{q}| .
\end{aligned}
$$

From definition (15), it appears clear that $\Phi(|\vec{x}|, t)$ defines the spatiotemporal retarded effects that the Gaussian white noise $\eta$ acting on the point $\vec{x}^{\prime}$ at time $t^{\prime}$ has on the probe in $\vec{x}$ at time $t$. The scaling of $\Phi(|\vec{x}|, t)$ for large and short times will be discussed in Section 2.2, resorting to its compact expression furnished in terms of the Fox $H$-functions [25]:

$$
\begin{aligned}
& \Phi(|\vec{x}|, t)=\frac{A^{\beta} K^{+} 2^{z-d}}{\pi^{d / 2}|\vec{x}|^{z}} \\
& \cdot H_{32}^{12}\left[\begin{array}{ccc}
2^{\gamma / 2} \frac{t}{\tau(|\vec{x}|)} & \left.\left.\begin{array}{cc}
(-\beta, 1) & \left(1-\frac{z}{2}, \frac{\gamma}{4}\right) \\
(-\beta, 1) & (0,1)
\end{array}\right)\left(\frac{d-z}{2}, \frac{\gamma}{4}\right)\right],
\end{array}\right.
\end{aligned}
$$

where the correlation time has been defined as $[24,25,43]$

$$
\tau(|\vec{x}|)=\frac{|\vec{x}|^{\gamma / 2}}{A} .
$$

The correlation properties of the fractional Gaussian noise (fGn) $\zeta(\vec{x}, t)$ are encapsulated in its $H$-function expression [44]

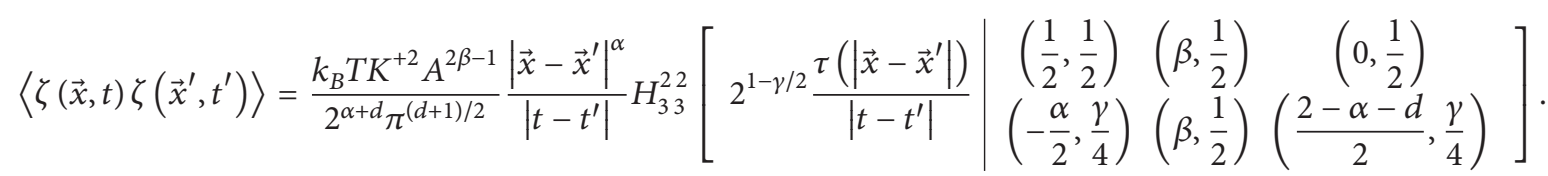

Setting $\vec{x} \rightarrow \vec{x}^{\prime}$ in (19), one recovers the (generalized) fluctuation-dissipation relation; that is,

$$
\left\langle\zeta(\vec{x}, t) \zeta\left(\vec{x}, t^{\prime}\right)\right\rangle=k_{B} T \frac{K^{+}}{\Gamma(1-\beta)\left|t-t^{\prime}\right|^{\beta}} .
$$

2.1. Correlation Functions. Any correlation function can be obtained within the FLE framework in (9) [44]. Moreover, the correlation functions can be casted in a compact and elegant expression by using the $H$-function formalism, which presents the practical advantage to study the limiting behaviours in a straightforward way. By instance, the position-position correlation function is expressed as [44]

$$
\begin{aligned}
& \left\langle h(\vec{x}, t) h\left(\vec{x}^{\prime}, t^{\prime}\right)\right\rangle \\
& =\frac{2 k_{B} T A^{2 \beta-1}}{2^{\alpha+d} \gamma \pi^{d / 2+1}}\left|\vec{x}-\vec{x}^{\prime}\right|^{\alpha} \int_{0}^{+\infty} \frac{\cos \left(\omega\left(t-t^{\prime}\right)\right)}{\omega^{2 \beta}} H_{13}^{21}\left[\begin{array}{c}
\left.\frac{\left(|\omega| \tau\left(\left|\vec{x}-\vec{x}^{\prime}\right|\right)\right)^{2 / \gamma}}{2} \mid \begin{array}{r}
\left(\beta, \frac{1}{\gamma}\right) \\
\left(\frac{-\alpha}{2}, \frac{1}{2}\right) \\
\left(\beta, \frac{1}{\gamma}\right)\left(\frac{2-\alpha-d}{2}, \frac{1}{2}\right)
\end{array}\right] d \omega .
\end{array}\right.
\end{aligned}
$$

We notice that this expression presents a divergence in the limit of $\omega \rightarrow 0$, as can be checked by expanding the Fox
$H$-function using the property (A.10). The same limit, however, allows extracting the position-position autocorrelation 
function:

$$
\begin{aligned}
\left\langle h(\vec{x}, t) h\left(\vec{x}, t^{\prime}\right)\right\rangle= & \frac{k_{B} T A^{\beta}}{2^{d-2} \pi^{d / 2} \gamma \Gamma(d / 2) \sin [\pi \alpha / \gamma]} \\
& \cdot \int_{0}^{+\infty} \frac{\cos \left(\omega\left(t-t^{\prime}\right)\right)}{\omega^{2 \beta+4 \alpha / \gamma}} d \omega .
\end{aligned}
$$

The divergence can be avoided by defining the time difference $\delta_{t} h(\vec{x}, t)=h(\vec{x}, t)-h(\vec{x}, 0)$, whose correlation function reads [43]

$$
\begin{aligned}
& \left\langle\delta_{t} h(\vec{x}, t) \delta_{t^{\prime}} h\left(\vec{x}^{\prime}, t^{\prime}\right)\right\rangle=\frac{k_{B} T}{(2 \pi)^{d / 2}}\left|\vec{x}-\vec{x}^{\prime}\right|^{z-d} \\
& .\left\{f\left[\frac{t}{\tau\left(\left|\vec{x}-\vec{x}^{\prime}\right|\right)}\right]+f\left[\frac{t^{\prime}}{\tau\left(\left|\vec{x}-\vec{x}^{\prime}\right|\right)}\right]\right. \\
& \left.-f\left[\frac{\left|t-t^{\prime}\right|}{\tau\left(\left|\vec{x}-\vec{x}^{\prime}\right|\right)}\right]\right\},
\end{aligned}
$$

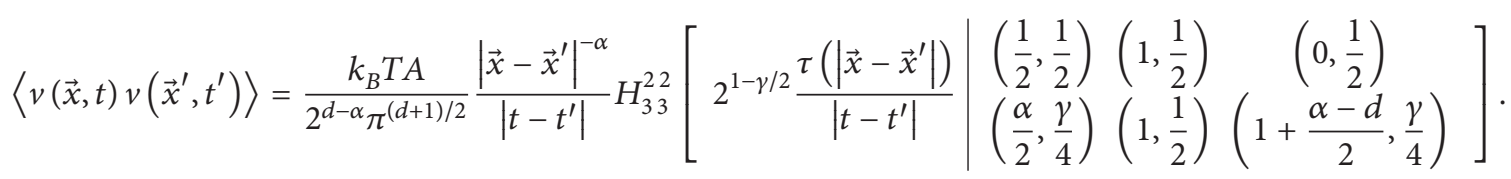

If we want to calculate the autocorrelation function we must set $\vec{x} \rightarrow \vec{x}^{\prime}$, achieving

$$
\begin{aligned}
& \left\langle v(\vec{x}, t) v\left(\vec{x}, t^{\prime}\right)\right\rangle \\
& =-\frac{\alpha k_{B} T A^{\beta} 2^{3-d}}{\gamma^{2} \pi^{d / 2}} \frac{\Gamma(1-\beta)}{\Gamma(d / 2)}\left|t-t^{\prime}\right|^{\beta-2},
\end{aligned}
$$

where $f[u]$ has been introduced in [43]. It is

(i) $f[u] \sim 2^{\alpha-d / 2} \frac{\Gamma(\alpha / 2)}{\Gamma((d-\alpha) / 2)} u$

(ii) $f[u] \sim \frac{2^{z+d / 2-2}}{\pi} z \sin \left(\frac{z \pi}{2}\right) \Gamma\left(\frac{z}{2}\right) \Gamma\left(\frac{z+d}{2}\right) u^{2}$

(iii) $f[u] \propto u^{\beta+1} e^{-u^{1 /(d-z)}}$

in the $u \ll 1$ regime, for (i) long range, (ii) local $(z \neq 2 m)$, and (iii) local $(z=2 m)$ hydrodynamic interactions, respectively. When $u \gg 1$ we have invariably

$$
f[u] \simeq \frac{2^{1-d / 2}}{z-d} \frac{\Gamma(1-\beta)}{\Gamma(d / 2)} u^{\beta} .
$$

The velocity correlation function gets the following expression:
Finally we report the Fox function expression for the position-velocity correlation function

thanks to (A.10).

$$
\left.\left\langle h(\vec{x}, t) v\left(\vec{x}^{\prime}, t^{\prime}\right)\right\rangle=\frac{k_{B} T A\left|\vec{x}-\vec{x}^{\star}\right|^{-\alpha}}{2^{d-\alpha} \pi^{d / 2}} H_{21}^{11}\left[A\left|t-t^{\prime}\right|\left(\frac{2}{\left|\vec{x}-\vec{x}^{\star}\right|}\right)^{\gamma / 2} \mid \begin{array}{c}
\left(1-\frac{\alpha}{2}, \frac{\gamma}{4}\right) \\
(0,1)
\end{array}\right] \begin{array}{c}
\left.\frac{d-\alpha}{2}, \frac{\gamma}{4}\right) \\
(0,1)
\end{array}\right]
$$

and its autocorrelation counterpart

$$
\left\langle h(\vec{x}, t) v\left(\vec{x}, t^{\prime}\right)\right\rangle=\frac{k_{B} T A^{\beta}}{\pi^{d / 2} 2^{d-2} \gamma} \frac{\Gamma(1-\beta)}{\Gamma(d / 2)}\left|t-t^{\prime}\right|^{\beta-1}
$$

2.2. $\Phi(|\vec{x}|, t)$ Scaling Behaviour. In this subsection we analyze the scaling behaviours of the noise-propagator $\Phi(|\vec{x}|, t)$ for times smaller and larger than the diffusion time $\tau$. Our analysis includes both long ranged and local hydrodynamic systems.

We start by considering the long range hydrodynamic systems. (i) Consider $t \ll \tau$. The $H$-function expression (17) can be expanded to the second order for small arguments according to (A.10), thus yielding

$$
\Phi(|\vec{x}|, t)
$$

$$
\simeq \frac{K^{+} A 2^{\gamma} \gamma}{\pi^{d / 2+1}} \sin \left(\frac{\pi \gamma}{4}\right) \frac{\Gamma((z+\alpha) / 2) \Gamma(\gamma / 4)}{\Gamma(1+2 \alpha / \gamma)} \frac{t^{2 \alpha / \gamma}}{|\vec{x}|^{z+\alpha}}
$$

The same result is obtained from expression (16). Indeed, by applying the following change of variable $y=(A t)^{2 / \gamma}|\vec{q}|$, 
the integral can be solved by the method furnished in Appendix B. In particular, thanks to (B.2), we have

$$
\begin{aligned}
& \Phi(|\vec{x}|, t) \\
& \simeq \frac{K^{+} A 2^{\alpha-d}}{\pi^{d / 2}} \Gamma\left(\frac{\alpha}{2}\right) \Gamma\left(\frac{d-\alpha}{2}\right)|\vec{x}|^{-\alpha}{ }_{0} D_{t}^{\beta} t^{\alpha / \gamma} .
\end{aligned}
$$

Recalling that the action of the Riemann fractional derivative on a power is given by [3]

$$
{ }_{0} D_{t}^{b} t^{a}=\frac{\Gamma(a+1)}{\Gamma(a+1-b)} t^{a-b}
$$

when $\Re e(a)>-1$ and $0<b<1$, we recover (30).

(ii) Consider $t \gg \tau$. Starting from (17) we expand to the second order the Fox function according to the rule in (A.11). The final expression for long times reads

$$
\begin{aligned}
\Phi(|\vec{x}|, t) \simeq & -\frac{K^{+} A^{-2 d / \gamma}(d-\alpha) 2^{3-d}}{\pi^{d / 2} \gamma^{2}} \\
& \cdot \frac{\sin (2 \pi(d-\alpha) / \gamma)}{\sin (2 \pi d / \gamma)} \frac{\Gamma(2(d-\alpha) / \gamma)}{\Gamma(d / 2)} \\
& \cdot t^{-2 z / \gamma} .
\end{aligned}
$$

We can obtain the same result studying the long time limit of the integral expression (16). Using the Laplace method for asymptotic integrals, as reported in Appendix C, we obtain that the integral in (16) can be solved:

$$
\begin{gathered}
\int_{0}^{+\infty}|\vec{q}|^{d / 2} J_{d / 2-1}(|\vec{q}||\vec{x}|) e^{-A|\vec{q}|^{\gamma / 2} t} d|\vec{q}| \\
\simeq \frac{A^{-2 d / \gamma} 2^{2-d}}{\gamma} \frac{\Gamma(2 d / \gamma)}{\Gamma(d / 2)} \frac{t^{-2 d / \gamma}}{|\vec{x}|^{1-d / 2}} .
\end{gathered}
$$

Plugging this result into (16) one gets

$$
\Phi(|\vec{x}|, t) \simeq \frac{K^{+} A^{-2 d / \gamma} 2^{2-d}}{\pi^{d / 2} \gamma} \frac{\Gamma(2 d / \gamma)}{\Gamma(d / 2)}{ }_{0} D_{t}^{\beta} t^{-2 d / \gamma}
$$

which coincides with (33) after (32) is applied.

We now turn to the study of systems with local hydrodynamic interactions.

(i) Consider $t \ll \tau$. We expand to the second order the $H$ function expression (17) taking advantage of (A.10):

$$
\begin{aligned}
& \Phi(|\vec{x}|, t) \\
& \simeq \frac{K^{+} A 2^{z-1} z}{\pi^{d / 2+1}} \sin \left(\frac{\pi z}{2}\right) \frac{\Gamma((d+z) / 2) \Gamma(z / 2)}{\Gamma((d+z) / z)} \frac{t^{d / z}}{|\vec{x}|^{d+z}}
\end{aligned}
$$

Performing the change of variable $y=(A t)^{1 / z}|\vec{q}|$ in (16) we solve the ensuing integral according to (B.2), recalling that $\alpha=d$ and $\gamma=2 z$ for systems characterized by local hydrodynamic interactions. Therefore we obtain

$$
\begin{aligned}
& \Phi(|\vec{x}|, t) \simeq \frac{K^{+} 2^{z-1} z}{\pi^{d / 2+1}} \\
& \cdot \sin \left(\frac{\pi z}{2}\right) \Gamma\left(\frac{d+z}{2}\right) \Gamma\left(\frac{z}{2}\right)|\vec{x}|^{-d-z}{ }_{0} D_{t}^{-\beta} t,
\end{aligned}
$$

which is reported to (36) once we apply (32).

(ii) Consider $t \gg \tau$. The long time limit expression for local hydrodynamic interactions can be achieved from the second order expansion (A.11). However a simple but tedious analysis reveals that different terms may lead the asymptotic expansion whether $z-d>2$ or $z-d<2$. In particular one has

$$
\Phi(|\vec{x}|, t) \simeq \begin{cases}\frac{K^{+} A^{-(2+d) / z} 2^{-d}}{\pi^{d / 2+1} z^{2}} \sin \left(\frac{2 \pi}{z}\right) \frac{\Gamma((2+d) / z) \Gamma((z-d-2) / z) \Gamma(2 / z)}{\Gamma((d+1) / 2)} \frac{t^{-(z+2) / z}}{|\vec{x}|^{-2}}, & z-d>2, \\ \frac{K^{+} A^{-1} 2^{1-z}}{\pi^{d / 2} z} \frac{\sin (\pi(z-d) / z)}{\sin (\pi(z-d) / 2)} \frac{\Gamma(\beta)}{\Gamma((z-d) / 2) \Gamma(z / 2)} \frac{t^{-(2 z-d) / z}}{|\vec{x}|^{d-z}}, & z-d<2 .\end{cases}
$$

The integral expression (16) can be handled to obtain the former result. Using Laplace's method outlined in Appendix $\mathrm{C}$, one has

$$
\Phi(|\vec{x}|, t) \simeq \frac{K^{+} A^{-d / z} 2^{1-d}}{\pi^{d / 2} z} \frac{\Gamma(d / z)}{\Gamma(d / 2)}{ }_{0} D_{t}^{\beta} t^{-2 d / z},
$$

which coincides with the first of (38), once we perform the Riemann-Liouville fractional derivative according to (32). However, the range of applicability of expression (32) is limited to systems satisfying the condition $z-d>2$. In order to reproduce the $z-d<2$ result, one has to handle expression (16) by applying the change of variables $y=|\vec{q}||\vec{x}|$, getting

$$
\begin{aligned}
& \Phi(|\vec{x}|, t) \\
& =\frac{K^{+}|\vec{x}|^{-d}}{(2 \pi)^{d / 2}} \int_{0}^{+\infty} y^{d / 2} J_{d / 2-1}(y)_{0} D_{t}^{\beta} e^{-y^{z}(t / \tau(|\vec{x}|))} d y .
\end{aligned}
$$

In Appendix D we calculate the long time limit of the fractional derivative applied to an exponential function. 
Hence, thanks to (D.4), we end up with the following equation:

$$
\begin{aligned}
& \Phi(|\vec{x}|, t) \\
& \simeq \frac{K^{+} A^{-1} 2^{-d / 2}}{\pi^{d / 2}} \frac{1}{\Gamma(-\beta)} \frac{t^{-1-\beta}}{|\vec{x}|^{d-z}} \int_{0}^{+\infty} y^{d / 2} J_{d / 2-1}(y) d y
\end{aligned}
$$

which corresponds to the second of (38), once the integral is solved [37].

The physical interpretation of the results obtained in this section can be explained as follows. Consider a tracer in $\vec{x}$ at time $t$ : the fGn acting on it is given by expression (15). From the former analysis it follows that the space-time roughly splits into two regions, $\left|\vec{x}-\vec{x}^{\prime}\right|>\left(A\left(t-t^{\prime}\right)\right)^{2 / \gamma}$ and $\left|\vec{x}-\vec{x}^{\prime}\right|<$ $\left(A\left(t-t^{\prime}\right)\right)^{2 / \gamma}$, where the propagator $\Phi\left(\left|\vec{x}-\vec{x}^{\prime}\right|, t-t^{\prime}\right)$ scaling behaviour is correspondingly different. Points lying within the first region are characterized by a propagator whose expressions are given by (30) or (36) whether the system presents long ranged or local hydrodynamic interactions, respectively. Roughly speaking, the contributions of these points to the fGn (15) are very small, asymptotically zero. To the contrary, the main contributions are given by the points in the second region, as it is apparent from (33) and (38). Remarkably, we notice that if the system is identified by local hydrodynamics, the contribution given by probes in the inner region $\left|\vec{x}-\vec{x}^{\prime}\right|<\left(A\left(t-t^{\prime}\right)\right)^{2 / \gamma}$ can be drastically different

$$
\Theta(|\vec{x}|, t)=\frac{A^{1+\beta} K^{+} 2^{\gamma / 2-d}}{\pi^{d / 2}|\vec{x}|^{\gamma / 2}} H_{32}^{12}\left[2^{\gamma / 2} \frac{\mathrm{t}}{\tau(|\vec{x}|)} \mid\right.
$$

$$
\left.\left(\begin{array}{c}
\left.1-\frac{\gamma}{4}, \frac{\gamma}{4}\right) \\
(0,1)
\end{array}\right)\left(\frac{2 d-z-\alpha}{2}, \frac{\gamma}{4}\right)\right]
$$

whether $z-d>2$ or $z-d<2$. This class of systems are usually termed Family-Vicsek $(z-d<2)$ [45] or superrough $(z-d>2)$ systems $[46,47]$, in correspondence with the different anomalous roughening properties.

\section{Fractional Langevin Equation with Applied Force}

We consider the case of a localized applied potential as expressed by the GEM constitutive equation (5). In [24, 43] it was shown that the FLE ruling the motion of a probe at a generic position $\vec{x}$ is

$$
\begin{aligned}
& K^{+}{ }_{-\infty} D_{t}^{\beta} \mathbf{h}(\vec{x}, t) \\
& =\int_{-\infty}^{t} \mathbf{F}\left\{\mathbf{h}\left(\vec{x}^{\star}, t^{\prime}\right), t^{\prime}\right\} \Theta\left(\left|\vec{x}-\vec{x}^{\star}\right|, t-t^{\prime}\right) d t^{\prime} \\
& \quad+\zeta(\vec{x}, t)
\end{aligned}
$$

where the force-propagator $\Theta(|\vec{x}|, t)$ has the same meaning of the force-propagator $\Phi(|\vec{x}|, t)$, representing the convolution kernel by which an external perturbation $\mathbf{F}\left\{\mathbf{h}\left(\vec{x}^{\star}, t^{\prime}\right), t^{\prime}\right\}$ exerted at the point $\vec{x}^{\star}$ at time $t^{\prime}$ is transferred to the point $\vec{x}$ at time $t$. The $H$-function expression of the force-propagator is [25] while the corresponding integral form reads

$$
\begin{aligned}
& \Theta(|\vec{x}|, t)=\frac{A K^{+}|\vec{x}|^{1-d / 2}}{(2 \pi)^{d / 2}} \\
& \quad \cdot{ }_{0} D_{t}^{\beta} \int_{0}^{+\infty}|\vec{q}|^{\alpha-d / 2} J_{d / 2-1}(|\vec{q}||\vec{x}|) e^{-A|\vec{q}|^{\gamma / 2} t} d|\vec{q}| .
\end{aligned}
$$

The fGn on the RHS of (42) satisfies the same correlation properties in (19). Finally, the FLE expression for the probe particle placed at $\vec{x}^{\star}$ is

$$
K^{+}{ }_{-\infty} D_{t}^{\beta} \mathbf{h}\left(\vec{x}^{\star}, t\right)=\mathbf{F}\left\{\mathbf{h}\left(\vec{x}^{\star}, t\right), t\right\}+\zeta\left(\vec{x}^{\star}, t\right) .
$$

3.1. $\Theta(|\vec{x}|, t)$ Scaling Behaviour. In analogy to Section 2.2, we study the scaling behaviours of the force-propagator in

the short and long time limits for long ranged and local hydrodynamic systems, respectively.

(i) Consider $t \ll \tau$. Expanding to the first order the $H$ function expression (43) by use of (A.10), we get

$$
\Theta(|\vec{x}|, t) \simeq \frac{K^{+} A 2^{\alpha}}{\pi^{d / 2}} \frac{\Gamma(\alpha / 2)}{\Gamma((d-\alpha) / 2) \Gamma(2 \alpha / \gamma)} \frac{t^{-\beta}}{|\vec{x}|^{\alpha}} .
$$

By applying the change of variable $y=(A t)^{2 / \gamma}|\vec{q}|$ to the integral in (44), making use of (B.4) and of the property (32) in sequence, we regain (46).

(ii) Consider $t \gg \tau$. Equation (A.11) allows the secondorder asymptotic expansion of (43). Two situations may arise according to whether the condition $z-d<2$ is satisfied or not: one has

$$
\Theta(|\vec{x}|, t) \simeq \begin{cases}\frac{K^{+} A^{1-2(2+\alpha) / \gamma} 2^{-d+2}}{\pi^{d / 2+1} \gamma^{2}} \frac{\Gamma(1-2(2+\alpha) / \gamma) \Gamma(2(2+\alpha) / \gamma) \Gamma(4 / \gamma)}{\Gamma((d+1) / 2)} \frac{t^{-1-4 / \gamma}}{|\vec{x}|^{-2}}, & z-d>2, \\ \frac{K^{+} 2^{2-z}}{\pi^{d / 2} \gamma} \frac{\sin (2 \pi(z-d) / \gamma)}{\sin (\pi(z-d) / 2)} \frac{\Gamma(\beta)}{\Gamma((z-d) / 2) \Gamma(z / 2)} \frac{t^{-1-\beta}}{|\vec{x}|^{d-z}}, & z-d<2 .\end{cases}
$$


It is possible to recover the same result studying the long time limit of the integral expression (44). Using the Laplace method for asymptotic integrals, as reported in Appendix C, we obtain that the integral in (44) can be solved:

$$
\begin{aligned}
& \int_{0}^{+\infty}|\vec{q}|^{\alpha-d / 2} J_{d / 2-1}(|\vec{q}||\vec{x}|) e^{-A|\vec{q}|^{\gamma / 2} t} d|\vec{q}| \\
& \quad \simeq-\frac{A^{-2(\alpha+2) / \gamma} 2^{2-d / 2}}{\gamma} \frac{\Gamma(2(2+\alpha) / \gamma)}{\Gamma(d / 2+1)} \frac{t^{-2(\alpha+2) / \gamma}}{|\vec{x}|^{-1-d / 2}} .
\end{aligned}
$$

Plugging this result into (44) and performing the time fractional derivative (32), we obtain the first of (47). The result for $z-d<2$ is recovered by first changing the variable $y=|\vec{q}||\vec{x}|$ in the integral appearing in (44); then the fractional derivative is applied to the exponential according to the rule (D.4), getting as intermediate result

$$
\begin{aligned}
& \Theta(|\vec{x}|, t) \\
& \simeq \frac{K^{+} 2^{-d / 2}}{\pi^{d / 2}} \frac{1}{\Gamma(-\beta)} \frac{t^{-1-\beta}}{|\vec{x}|^{d-z}} \int_{0}^{+\infty} y^{d / 2-z} J_{d / 2-1}(y) d y .
\end{aligned}
$$

Finally, performing the remaining integral [37] we get the second of (47).

The scaling behaviour of the local hydrodynamic force-propagator can be inferred from the corresponding behaviour of the force-propagator. As a matter of fact both expressions coincide but for a constant factor $A$, as it is immediately apparent by a direct comparison of (16) and (44).

Any perturbation exerted on the tagged probe is therefore transferred to a generic probe placed in $|\vec{x}|$ by the action of $\Theta(|\vec{x}|, t)$. As in the case of $\Phi(|\vec{x}|, t)$, however, the contributions are very different if the probes' distance is such that $\left|\vec{x}-\vec{x}^{*}\right|>\left(A\left(t-t^{\prime}\right)\right)^{2 / \gamma}$ or $\left|\vec{x}-\vec{x}^{*}\right|<\left(A\left(t-t^{\prime}\right)\right)^{2 / \gamma}$. In particular we notice that, in case of long ranged hydrodynamics, if the tagged probe is in the inner region $\left|\vec{x}-\vec{x}^{*}\right|<(A(t-$ $\left.\left.t^{\prime}\right)\right)^{2 / \gamma}$, its influence on the motion of the untagged probe might be considerably different according to whether we are considering Family-Vicsek or superrough systems (see (47)). This contrasts with the noise-propagator expression (33), where the strength of the hydrodynamic interactions appearing in the FD relation (2) suppresses any elastic effect inherent to the system dynamics.

\section{Kubo Fluctuation Relations}

The Kubo fluctuation relation (KFR) expresses the mean response of the variable $X_{i}$ at time $t$ to a perturbation applied to a variable $X_{j}$ at time $0[24,25,48-50]$. If the dynamics of the system is given, for instance, in the form $d \mathbf{X} / d t=\mathbf{f}(\mathbf{X})$, the mean linear response of the $i$ th degree of freedom to a small perturbation of the $j$ th component of the vector field $f_{j} \rightarrow f_{j}+\delta f_{j}$ can be expressed as

$$
\left\langle X_{i}(t)\right\rangle=\frac{1}{k_{B} T} \int_{-\infty}^{t}\left\langle X_{i}(t-s) \dot{X}_{j}(0)\right\rangle \delta f_{j}(s) d s
$$

where $\left\langle X_{i}(t-s) \dot{X}_{j}(0)\right\rangle$ represents the two-time correlation computed in the unperturbed system.
When the perturbation consists in a constant force $F_{0}$ applied along one direction (say $F_{0}=F_{j}$ ), we can perform the following substitution in (5), (42), and (45):

$$
\mathbf{F}\left\{\mathbf{h}\left(\vec{x}^{\star}, t\right), t\right\}=F_{0} \theta(t)
$$

In $[25,43]$ we have studied the average drift of the untagged and tagged probes to such an external force, that is, $\langle h(\vec{x}, t)\rangle_{F_{0}}$ and $\left\langle h\left(\vec{x}^{\star}, t\right)\right\rangle_{F_{0}}$, showing by a direct computation that KFR (50) is exactly fulfilled. In particular we have demonstrated the following equalities:

$$
\begin{aligned}
& \langle h(\vec{x}, t)\rangle_{F_{0}}=\frac{F_{0}}{2 k_{B} T}\left\langle\delta_{t} h(\vec{x}, t) \delta_{t} h\left(\vec{x}^{\star}, t\right)\right\rangle, \\
& \langle h(\vec{x}, t)\rangle_{F_{0}}=\frac{F_{0}}{k_{B} T} \int_{0}^{t}\left\langle h(\vec{x}, t) v\left(\vec{x}^{\star}, 0\right)\right\rangle d t^{\prime},
\end{aligned}
$$

where the unperturbed correlation functions are given in (23) and (28), respectively. Notice that the KFR in presence of a constant force coincides with the Generalized Einstein Relation (GER). In the following section, we demonstrate the validity of the KFR in case of an applied perturbation of the type

$$
\mathbf{F}\left\{\mathbf{h}\left(\vec{x}^{\star}, t\right), t\right\}=F_{0} \cos \left(\omega_{0} t\right) .
$$

4.1. Time Periodic Force. Upon applying the time periodic force (53) to the tagged probe, FLEs (42), (45) take the form

$$
\begin{aligned}
& K^{+}{ }_{-\infty} D_{t}^{\beta} h(\vec{x}, t) \\
& =F_{0} \Re e\left[\int_{-\infty}^{+\infty} e^{-i \omega_{0} t^{\prime}} \Theta\left(\left|\vec{x}-\vec{x}^{\star}\right|, t-t^{\prime}\right) d t^{\prime}\right] \\
& \quad+\zeta(\vec{x}, t), \\
& K^{+} D_{t}^{\beta} h\left(\vec{x}^{\star}, t\right)=F_{0} \Re e\left[e^{-i \omega_{0} t}\right]+\zeta\left(\vec{x}^{\star}, t\right) .
\end{aligned}
$$

Our attention is on the average drifts $\langle v(\vec{x}, t)\rangle_{F_{0}}=d\langle h(\vec{x}$, $t)\rangle_{F_{0}} / d t$ and $\left\langle v\left(\vec{x}^{\star}, t\right)\right\rangle_{F_{0}}=d\left\langle h\left(\vec{x}^{\star}, t\right)\right\rangle_{F_{0}} / d t$; hence it is useful to firstly consider the complex positions $\langle H(\vec{x}, t)\rangle_{F_{0}}$ and $\left\langle H\left(\vec{x}^{\star}, t\right)\right\rangle_{F_{0}}$ to get the real quantities afterwards as $\langle h(\vec{x}, t)\rangle_{F_{0}}$ $=\mathfrak{R} e[H(\vec{x}, t)]$ and $\left\langle h\left(\vec{x}^{\star}, t\right)\right\rangle_{F_{0}}=\mathfrak{R} e\left[H\left(\vec{x}^{\star}, t\right)\right]$. The FLEs ruling out the dynamics of the complex positions are

$$
\begin{aligned}
& K^{+}{ }_{-\infty} D_{t}^{\beta} H(\vec{x}, t) \\
& \quad=F_{0} \int_{-\infty}^{+\infty} e^{-i \omega_{0} t^{\prime}} \Theta\left(\left|\vec{x}-\vec{x}^{\star}\right|, t-t^{\prime}\right) d t^{\prime}+\zeta(\vec{x}, t),
\end{aligned}
$$

$$
K^{+}{ }_{-\infty} D_{t}^{\beta} H\left(\vec{x}^{\star}, t\right)=F_{0} e^{-i \omega_{0} t}+\zeta\left(\vec{x}^{\star}, t\right) .
$$


Thus performing the Fourier transform in time and taking the average values one readily obtains

$$
\begin{aligned}
& \langle H(\vec{x}, t)\rangle_{F_{0}}=\frac{A\left|\vec{x}-\vec{x}^{\star}\right|^{1-d / 2}}{(2 \pi)^{d / 2}} F_{0} \int_{0}^{+\infty}|\vec{q}|^{\alpha-d / 2} \\
& \cdot J_{d / 2-1}\left(|\vec{q}|\left|\vec{x}-\vec{x}^{\star}\right|\right) \frac{e^{-i \omega_{0} t}}{-i \omega_{0}+A|\vec{q}|^{\gamma / 2}} d|\vec{q}|, \\
& \left\langle H\left(\vec{x}^{\star}, t\right)\right\rangle_{F_{0}}=\frac{2^{2-d} A^{\beta}}{\gamma(2 \pi)^{d / 2} \Gamma(d / 2) \sin (\pi \beta)} F_{0} \\
& \cdot \frac{e^{-i \omega_{0} t}}{\left(-i \omega_{0}\right)^{\beta}},
\end{aligned}
$$

where we explicitly reported the Fourier transform of the force-propagator expression (44). The complex velocities are achieved by performing the time derivative of the complex positions and can be casted in the following form:

$$
\begin{aligned}
\langle V(\vec{x}, t)\rangle_{F_{0}} & =\mu\left(\vec{x}, \omega_{0}\right) F_{0} e^{-i \omega_{0} t}, \\
\left\langle V\left(\vec{x}^{\star}, t\right)\right\rangle_{F_{0}} & =\mu\left(\vec{x}^{\star}, \omega_{0}\right) F_{0} e^{-i \omega_{0} t},
\end{aligned}
$$

owing to the definitions of the complex mobility or admittance

$$
\mu\left(\vec{x}, \omega_{0}\right)=\frac{A\left|\vec{x}-\vec{x}^{\star}\right|^{1-d / 2}}{(2 \pi)^{d / 2}} \int_{0}^{+\infty}|\vec{q}|^{\alpha-d / 2}
$$

$$
\left.\begin{array}{l}
\left\langle v\left(\vec{x}, \omega_{0}\right) v\left(\vec{x}^{\star}, \omega_{0}^{\prime}\right)\right\rangle \\
=\frac{k_{B} T A^{2 \beta-1}}{2^{\alpha+d-2} \gamma \pi^{d / 2-1}}\left|\vec{x}-\vec{x}^{\star}\right|^{\alpha}\left|\omega_{0}\right|^{2-2 \beta} \delta\left(\omega_{0}+\omega_{0}^{\prime}\right) H_{13}^{21}\left[\frac{\left[\omega_{0} \tau\left(\left|\vec{x}-\vec{x}^{\star}\right|\right)\right]^{\gamma / 2}}{2} \mid\left(-\frac{\alpha}{2}, \frac{1}{2}\right)\left(\beta, \frac{1}{\gamma}\right)\right. \\
\left(\beta, \frac{1}{\gamma}\right)
\end{array}\right] .
$$
in time of the velocity correlation function in (26) reads [25]

To complete the demonstration of the validity of the KFR also in presence of a time oscillatory force, we need to relate the complex mobility to the velocity correlation function in absence of perturbations. This is done according to the Green-Kubo relation that connects the transport coefficients to the equilibrium two-time correlations of a system:

$$
\begin{aligned}
& \left\langle v\left(\vec{x}, \omega_{0}\right) v\left(\vec{x}^{\star}, \omega_{0}^{\prime}\right)\right\rangle \\
& \quad=4 \pi \delta\left(\omega_{0}+\omega_{0}^{\prime}\right) k_{B} T \Re e\left[\mu\left(\vec{x}, \omega_{0}\right)\right] .
\end{aligned}
$$

By using the properties (A.5), (A.2), and (A.6) in sequence, one arrives at

$$
\begin{aligned}
& \left\langle v\left(\vec{x}, \omega_{0}\right) v\left(\vec{x}^{\star}, \omega_{0}^{\prime}\right)\right\rangle \\
& \quad=\frac{k_{B} T A}{2^{d-\alpha-1} \pi^{d / 2-1}}\left|\vec{x}-\vec{x}^{\star}\right|^{-\alpha} \delta\left(\omega_{0}+\omega_{0}^{\prime}\right) H_{31}^{12}\left[\frac{2^{\gamma / 2}}{\omega_{0} \tau\left(\left|\vec{x}-\vec{x}^{\star}\right|\right)} \mid \begin{array}{c}
\left(1-\frac{\alpha}{2}, \frac{\gamma}{4}\right)\left(0, \frac{1}{2}\right)\left(\frac{d-\alpha}{2}, \frac{\gamma}{4}\right) \\
\left(0, \frac{1}{2}\right)
\end{array}\right] .
\end{aligned}
$$

We now take the complex mobility in (58) and evaluate its $\mathrm{H}$-function expression. Applying the change of variable
$y=\left(A / \omega_{0}\right)^{2 / \gamma}|\vec{q}|$ we obtain for its real and imaginary parts 


$$
\begin{aligned}
& \mathfrak{R} e\left[\mu\left(\vec{x}, \omega_{0}\right)\right]=\frac{A\left|\vec{x}-\vec{x}^{\star}\right|^{1-d / 2}}{(2 \pi)^{d / 2}}\left(\frac{\omega_{0}}{A}\right)^{(2+\alpha-d) / \gamma} \\
& \cdot \int_{0}^{+\infty} \frac{y^{\alpha-d / 2}}{1+y^{\gamma}} J_{d / 2-1}\left(y\left(\omega_{0} \tau\left(\left|\vec{x}-\vec{x}^{\star}\right|\right)\right)^{2 / \gamma}\right) d y, \\
& \Im m\left[\mu\left(\vec{x}, \omega_{0}\right)\right] \\
& =\frac{A^{2}\left|\vec{x}-\vec{x}^{\star}\right|^{1-d / 2}}{(2 \pi)^{d / 2} \omega_{0}}\left(\frac{\omega_{0}}{A}\right)^{(2 z+4 \alpha-3 d+1) / \gamma} \\
& \cdot \int_{0}^{+\infty} \frac{y^{z+2 \alpha-3 d / 2}}{1+y^{\gamma}} J_{d / 2-1}\left(y\left(\omega_{0} \tau\left(\left|\vec{x}-\vec{x}^{\star}\right|\right)\right)^{2 / \gamma}\right) d y .
\end{aligned}
$$

Then, recalling that $1 /\left(1+y^{\delta}\right)=(1 / \delta) H_{11}^{11}\left[y \mid \begin{array}{c}(1,1 / \delta) \\ (1,1 / \delta)\end{array}\right][44]$, it follows that

$$
\begin{gathered}
\mathfrak{R} e\left[\mu\left(\vec{x}, \omega_{0}\right)\right]=\frac{A\left|\vec{x}-\vec{x}^{\star}\right|^{1-d / 2}}{(2 \pi)^{d / 2} \gamma}\left(\frac{\omega_{0}}{A}\right)^{(2+\alpha-d) / \gamma} \\
\cdot \int_{0}^{+\infty} y^{\alpha-d / 2} J_{d / 2-1}\left(y\left(\omega_{0} \tau\left(\left|\vec{x}-\vec{x}^{\star}\right|\right)\right)^{2 / \gamma}\right)
\end{gathered}
$$

$$
\begin{aligned}
& \cdot H_{11}^{11}\left[y \mid \begin{array}{l}
\left(1, \frac{1}{\gamma}\right) \\
\left(1, \frac{1}{\gamma}\right)
\end{array}\right] d y, \\
& \Im m\left[\mu\left(\vec{x}, \omega_{0}\right)\right] \\
& =\frac{A^{2}\left|\vec{x}-\vec{x}^{\star}\right|^{1-d / 2}}{(2 \pi)^{d / 2} \omega_{0} \gamma}\left(\frac{\omega_{0}}{A}\right)^{(2 z+4 \alpha-3 d+1) / \gamma} \\
& \cdot \int_{0}^{+\infty} y^{z+2 \alpha-3 d / 2} J_{d / 2-1}\left(y\left(\omega_{0} \tau\left(\left|\vec{x}-\vec{x}^{\star}\right|\right)\right)^{2 / \gamma}\right) \\
& \cdot H_{11}^{11}\left[\begin{array}{l|l}
\left(1, \frac{1}{\gamma}\right) \\
\left(1, \frac{1}{\gamma}\right)
\end{array}\right] d y .
\end{aligned}
$$

We apply the properties (A.7) and (A.5) to $\mathfrak{R} e\left[\mu\left(\vec{x}, \omega_{0}\right)\right]$ and (A.7), (A.5), and (A.6) to $\Im m\left[\mu\left(\vec{x}, \omega_{0}\right)\right]$; then we get the sought expressions:

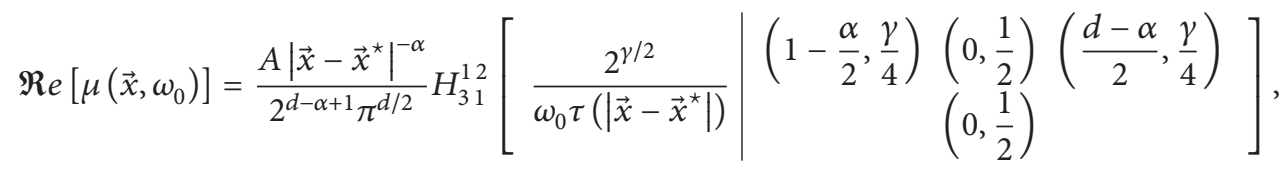

$$
\begin{aligned}
& \Im m\left[\mu\left(\vec{x}, \omega_{0}\right)\right]=\frac{A\left|\vec{x}-\vec{x}^{\star}\right|^{-\alpha}}{2^{d-\alpha+1} \pi^{d / 2}} H_{31}^{12}\left[\frac{2^{\gamma / 2}}{\omega_{0} \tau\left(\left|\vec{x}-\vec{x}^{\star}\right|\right)} \mid \begin{array}{c}
\left(1-\frac{\alpha}{2}, \frac{\gamma}{4}\right) \\
\left(\frac{1}{2}, \frac{1}{2}\right) \\
\left(\frac{1}{2}, \frac{1}{2}\right)
\end{array}\right] .
\end{aligned}
$$

The Green-Kubo relation (60) follows by comparison of the real part of (65) with (62).

4.2. Complex Admittance Scaling Behaviour. Thanks to the short and long term expansion of the Fox $H$-functions, we can study the high and low frequency limits of the complex admittance $\mu\left(\vec{x}, \omega_{0}\right)$. We do it by considering the case of long range hydrodynamics and local one, respectively.

(i) Consider $\omega_{0} \ll \tau^{-1}$. In the low frequency limit the property (A.11) entails

$$
\begin{aligned}
& \mathfrak{R} e\left[\mu\left(\vec{x}, \omega_{0}\right)\right] \simeq \frac{\sin (\pi \beta / 2)}{K^{+}} \omega_{0}^{1-\beta}, \\
& \Im m\left[\mu\left(\vec{x}, \omega_{0}\right)\right] \simeq \frac{\cos (\pi \beta / 2)}{K^{+}} \omega_{0}^{1-\beta} .
\end{aligned}
$$

We point out that the same result is easily obtained by handling the mobility integral representation (63) [24]. The former result can be casted in the following compact form:

$$
\mu\left(\vec{x}, \omega_{0}\right) \simeq \frac{\omega_{0}^{1-\beta}}{K^{+}} e^{-i(1-\beta)(\pi / 2)},
$$

which has a simple physical interpretation on the basis of (59). It means that the response signal is amplified by a factor $\left|\mu\left(\vec{x}, \omega_{0}\right)\right|=\omega_{0}^{1-\beta} / K^{+}$with respect the input signal, and it presents a constant phase shift equal to $\varphi\left(\vec{x}, \omega_{0}\right)=(1-$ $\beta)(\pi / 2)$. The fact that both amplitude and phase are independent of the distance $\left|\vec{x}-\vec{x}^{\star}\right|$ is worth mentioning, since it signifies that no difference exists between the behaviour of a generic probe and the tagged one's.

(ii) Consider $\omega_{0} \gg \tau^{-1}$. By adopting the expansion (A.10) we gain

$$
\begin{aligned}
& \mathfrak{R} e\left[\mu\left(\vec{x}, \omega_{0}\right)\right] \simeq \frac{A\left|\vec{x}-\vec{x}^{\star}\right|^{-\alpha}}{2^{d-\alpha} \pi^{d / 2}} \frac{\Gamma(\alpha / 2)}{\Gamma((d-\alpha) / 2)}, \\
& \Im m\left[\mu\left(\vec{x}, \omega_{0}\right)\right] \\
& \simeq \frac{A\left|\vec{x}-\vec{x}^{\star}\right|^{-\alpha}}{2^{2(d-\alpha)-z} \pi^{d / 2} \omega_{0} \tau\left(\left|\vec{x}-\vec{x}^{\star}\right|\right)} \frac{\Gamma((z+2 \alpha-d) / 2)}{\Gamma((2(d-\alpha)-z) / 2)} .
\end{aligned}
$$

The phase shift can be calculated as

$$
\tan \varphi\left(\vec{x}, \omega_{0}\right) \simeq-\frac{2^{2 d-3 \alpha-z}}{\pi}
$$




$$
\begin{aligned}
& \cdot \frac{\Gamma((d-\alpha) / 2) \Gamma((z-d) / 2+\alpha)}{\Gamma(\alpha / 2) \Gamma((2(d-\alpha)-z) / 2)} \\
& \cdot \frac{1}{\omega_{0} \tau\left(\left|\vec{x}-\vec{x}^{\star}\right|\right)} .
\end{aligned}
$$

Therefore in the large $\omega_{0} \tau$ limit the imaginary part is negligible $\left(\varphi\left(\vec{x}, \omega_{0}\right) \rightarrow 0\right)$. It follows that the response amplitude is dominated by the real part which has no frequency dependence.

We now consider the scaling behaviour of $\mu\left(\vec{x}, \omega_{0}\right)$ for systems presenting local hydrodynamics interactions.

(i) Consider $\omega_{0} \ll \tau^{-1}$. The leading term in (65) yields

$$
\begin{aligned}
& \Re e\left[\mu\left(\vec{x}, \omega_{0}\right)\right] \simeq \frac{\sin (\pi \beta / 2)}{K^{+}} \omega_{0}^{1-\beta}, \\
& \Im m\left[\mu\left(\vec{x}, \omega_{0}\right)\right] \simeq \frac{\cos (\pi \beta / 2)}{K^{+}} \omega_{0}^{1-\beta},
\end{aligned}
$$

in analogy with the behaviour observed in long range hydrodynamics systems in the same limit (66).

(ii) Consider $\omega_{0} \gg \tau^{-1}$. The second-order term in the expansion (A.10) gives for $z \neq m$

$$
\begin{aligned}
& \mathfrak{R} e\left[\mu\left(\vec{x}, \omega_{0}\right)\right] \simeq \frac{A 2^{2 z}}{\pi^{d / 2+1}} z \\
& \cdot \sin (z \pi) \Gamma\left(\frac{d}{2}+z\right) \Gamma(z) \frac{\left(\omega_{0} \tau\left(\left|\vec{x}-\vec{x}^{\star}\right|\right)\right)^{-2}}{\left|\vec{x}-\vec{x}^{\star}\right|^{d}} \\
& \Im \mathfrak{m}\left[\mu\left(\vec{x}, \omega_{0}\right)\right] \simeq \frac{A 2^{z-1}}{\pi^{d / 2-1}} z \\
& \cdot \sin \left(\frac{z \pi}{2}\right) \Gamma\left(\frac{d+z}{2}\right) \Gamma\left(\frac{z}{2}\right) \frac{\left(\omega_{0} \tau\left(\left|\vec{x}-\vec{x}^{\star}\right|\right)\right)^{-1}}{\left|\vec{x}-\vec{x}^{\star}\right|^{d}}
\end{aligned}
$$

from which it turns out that the phase is

$$
\begin{aligned}
& \tan \varphi\left(\vec{x}, \omega_{0}\right) \\
& \quad \simeq-2^{-z-2} \frac{\Gamma((d+z) / 2) \Gamma(z / 2)}{\Gamma(d / 2+z) \Gamma(z)} \frac{\omega_{0} \tau\left(\left|\vec{x}-\vec{x}^{\star}\right|\right)}{\cos (z \pi / 2)} .
\end{aligned}
$$

In the very high frequency limit then the phase shift is $\varphi\left(\vec{x}, \omega_{0}\right) \sim-\pi / 2$ if $4 m<z<4 m+1$ or $4 m+3<z<4 m+4$ and $\varphi\left(\vec{x}, \omega_{0}\right) \sim \pi / 2$ if $4 m+1<z<4 m+2$. As a consequence, the response amplitude is given by $\left|\mu\left(\vec{x}, \omega_{0}\right)\right| \simeq\left|\Im m\left[\mu\left(\vec{x}, \omega_{0}\right)\right]\right|$.

The case $z=m$ is analytically very hard to tackle, since there is no expansion of $H$-function work in this case nor the integral expression (63) can help. Nevertheless we can say that $\left|\mu\left(\vec{x}, \omega_{0}\right)\right|$ is exponentially small and $\varphi\left(\vec{x}, \omega_{0}\right) \simeq(1-\beta)(\pi / 2)-$ $\left(\omega_{0} \tau\right)^{1 / z}$ : for instance, the exact solution for $z=2, d=1$ reads $\mu\left(\vec{x}, \omega_{0}\right)=\left(\sqrt{\omega_{0} A} / 2\right) e^{-\sqrt{\omega_{0} / 2 A}\left|\vec{x}-\vec{x}^{\star}\right|} e^{-i\left(\pi / 4-\sqrt{\omega_{0} / 2 A}\left|\vec{x}-\vec{x}^{\star}\right|\right)}$ (see Section 4.3 for a practical example).

The physical scenario emerging from this analysis can be illustrated with the help of Figure 1. Let us say that we perturb the system at a frequency $\omega_{0}$ : thanks to definition (18) the system splits into two spatial regions, $\left|\vec{x}-\vec{x}^{\star}\right|<\left(A / \omega_{0}\right)^{2 / \gamma}$ (I) and $\left|\vec{x}-\vec{x}^{\star}\right|>\left(A / \omega_{0}\right)^{2 / \gamma}$ (II), characterized by well distinct dynamical phases. For long-range or local hydrodynamics, the response of the inner region (I), that is, that closer to the tagged probe, shows a universal dependence $\propto \omega_{0}^{1-\beta}$ and a phase shift $(1-\beta) / 2$ with respect to the applied oscillatory force. On the other hand, in the outer region (II) in long ranged hydrodynamic systems, the response amplitude decays as $\left|\vec{x}-\vec{x}^{\star}\right|^{-\alpha}$, but almost no phase delay is displayed when compared to the external force. When the hydrodynamic interactions are local, the amplitude of the response is smaller and decays faster, namely, $\propto \mid \vec{x}-$ $\left.\vec{x}^{\star}\right|^{-d-z} \omega_{0}^{-1}$ for $z \neq 2 m$ and $\propto e^{-\left|\vec{x}-\vec{x}^{\star}\right| \omega_{0}^{1 / z}}$ for $z=2 m$; the phase shift instead is $\varphi\left(\vec{x}, \omega_{0}\right) \sim \pm \pi / 2$ when $z=2 \mathrm{~m}$, and it grows like $\varphi\left(\vec{x}, \omega_{0}\right) \sim-\omega_{0}^{1 / z}\left|\vec{x}-\vec{x}^{\star}\right|$ if $z=2 m$.

4.3. Single File Systems. From [39] we know that the effective equation for the particle position $h(x, t)$ in single file systems with applied periodic force is given by

$$
\begin{aligned}
\xi \frac{\partial}{\partial t} h(x, t)= & \kappa \frac{\partial^{2}}{\partial x^{2}} h(\vec{x}, t)+F_{0} \cos \left(\omega_{0} t\right) \delta\left(x^{\star}-x\right) \\
& +\eta(\vec{x}, t) .
\end{aligned}
$$

In (73) $\xi$ represents the damping term and $\kappa=\rho^{2} k_{B} T$ with $\rho$ file's density. Hereafter we will choose $\kappa=1$. Therefore, to connect our previous analysis with the present system we set $A=1 / \xi, \alpha=d=1$, and $z=2$, which in turn gives $K^{+}=2 \sqrt{\xi}$ and $\beta=1 / 2$ from (11) and (10), respectively. According to (55) the complex FLEs for tagged and untagged probe are

$$
\begin{aligned}
& 2 \sqrt{\xi} D_{C}^{1 / 2} H(x, t) \\
& =F_{0} \int_{-\infty}^{+\infty} e^{-i \omega_{0} t^{\prime}} \Theta\left(\left|x-x^{\star}\right|, t-t^{\prime}\right) d t^{\prime}+\zeta(x, t), \\
& 2 \sqrt{\xi} D_{C}^{1 / 2} H\left(x^{\star}, t\right)=F_{0} e^{-i \omega_{0} t}+\zeta\left(x^{\star}, t\right) .
\end{aligned}
$$

We recall that the internal coordinate $x$ in single file systems has the meaning of the ordering number of the particle along the file $[1,39,43,44]$. We study the average position of the $x$ th particle (untagged probe) by taking the real part of the complex drifts (56):

$$
\begin{aligned}
& \langle h(x, t)\rangle_{F_{0}}=\frac{F_{0} \xi}{\pi} \int_{0}^{\infty} \frac{\cos \left(q\left|x-x^{\star}\right|\right)}{\xi^{2} \omega_{0}^{2}+q^{4}}\left(\frac{q^{2}}{\xi} \cos \left(\omega_{0} t\right)\right. \\
& \left.\quad+\omega_{0} \sin \left(\omega_{0} t\right)\right) d q .
\end{aligned}
$$

The two integrals can be solved explicitly [51], so that we obtain after straightforward passages

$$
\begin{aligned}
\langle h(x, t)\rangle_{F_{0}}= & \frac{F_{0}}{2 \sqrt{\omega_{0} \xi}} e^{-\left|x-x^{\star}\right| \sqrt{\omega_{0} \xi / 2}} \\
& \cdot \cos \left(\omega_{0} t-\left|x-x^{\star}\right| \sqrt{\frac{\omega_{0} \xi}{2}}-\frac{\pi}{4}\right) .
\end{aligned}
$$




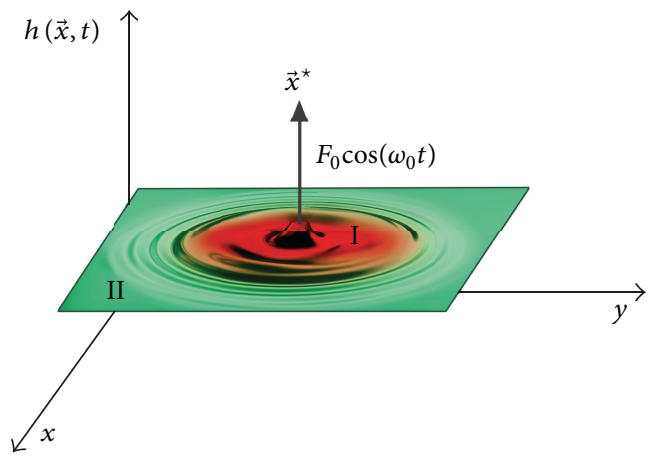

(a)

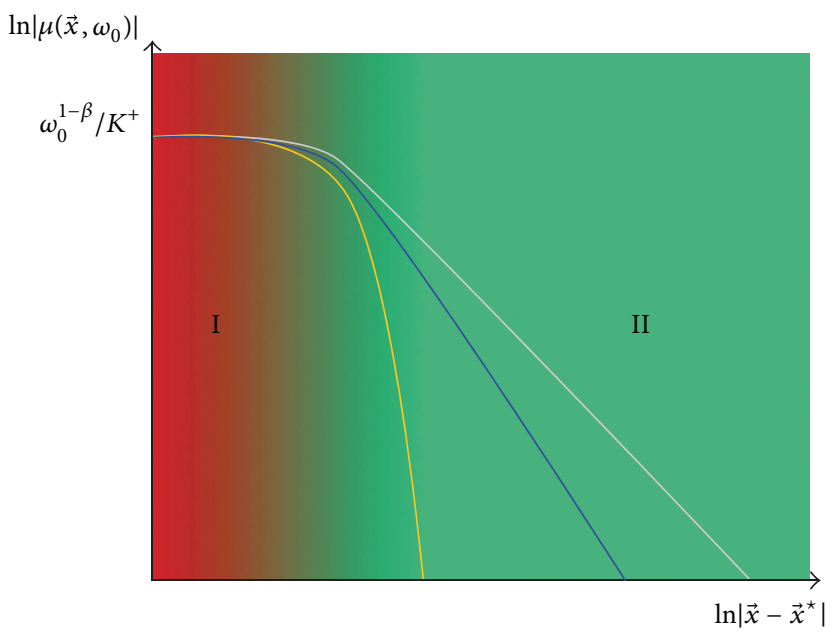

(b)

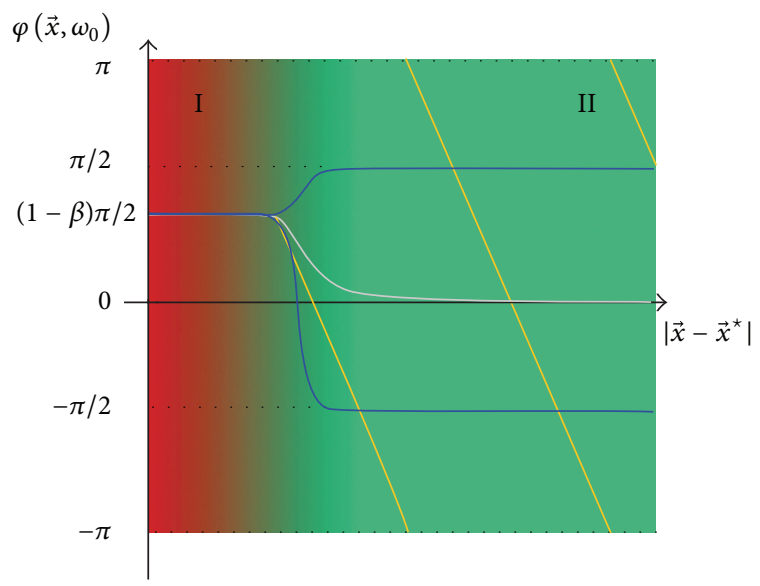

(c)

Figure 1: (Color online) Time periodic force $F_{0} \cos \left(\omega_{0} t\right)$. (a) 3D rendering of a membrane described by (1), under the effect of an applied time periodic force in $\vec{x}^{\star}$ (black arrow). $h(\vec{x}, t)$ represents the height of the fluctuating membrane on a 2-dimensional substrate $(\vec{x}=(x, y))$. Regions I and II correspond, respectively, to the inner and outer region in which the membrane separates when the force is applied. The color code, red for region I and green for region II, has been drawn for the reader's convenience: increasing the frequency $\omega_{0}$ entails the shrinkage of the red region (I). (b) Schematic representation of the untagged response amplitude $\left|\mu\left(\vec{x}, \omega_{0}\right)\right|$ as a function of the distance $\left|\vec{x}-\vec{x}^{\star}\right|$ : since it is a schematic drawing no scale is needed on the $x$-axis. In region I the universal behaviour $\omega_{0}^{1-\beta} / K^{+}$holds for any kind of hydrodynamic interactions. In region II the decay of the response's amplitude is $\propto\left|\vec{x}-\vec{x}^{\star}\right|^{-\alpha}$ for long range hydrodynamic systems (grey (upper) solid line), $\propto\left|\vec{x}-\vec{x}^{\star}\right|^{-z-d}$ for local hydrodynamic systems with $z \neq 2 m$ with $m \in \mathbb{N}$ (blue (middle) solid line), and exponentially fast for local hydrodynamic systems with $z=2 m$ (orange (bottom) line). (c) Schematic representation of the untagged response phase $\varphi\left(\vec{x}, \omega_{0}\right)$ as a function of the distance $\left|\vec{x}-\vec{x}^{\star}\right|$. No scale is needed on the $x$-axis. Region I: the system displays a universal phase delay for long range and local hydrodynamic interactions; that is, $\varphi\left(\vec{x}, \omega_{0}\right)=(1-\beta)(\pi / 2)$. Region II: for long range hydrodynamic systems the phase is absent; that is, $\varphi\left(\vec{x}, \omega_{0}\right) \simeq 0$ (grey (middle) solid line); for local hydrodynamics the phase is approximately $\pi / 2$ if $1+4 m<z<3+4 m$ with $m \in \mathbb{N}$ (upper blue line), while it is approximately $-\pi / 2$ if $4 m<z<1+4 m$ or $3+4 m<z<4+4 m$ (bottom blue line); if the hydrodynamic interactions are local and $z=2 m$ the phase shows a linear dependence on the distance $\left|\vec{x}-\vec{x}^{\star}\right|$; that is, $\varphi\left(\vec{x}, \omega_{0}\right) \sim-\left|\vec{x}-\vec{x}^{\star}\right|$ (orange (bright linear) solid line).

For the $x^{\star}$ th tagged particle we easily find

$$
\left\langle h\left(x^{\star}, t\right)\right\rangle_{F_{0}}=\frac{F_{0}}{2 \sqrt{\omega_{0} \xi}} \cos \left(\omega_{0} t-\frac{\pi}{4}\right) .
$$

The expression (77) reproduces the formula given in [39] for the tagged tracer. We checked these results by numerical simulations: a file of $N$ Brownian point-like particles moves on a ring of length $L(\rho=N / L)$, while the particle $x^{\star}$ is subjected to a periodic force $F_{0} \cos \left(\omega_{0} t\right)$. In Figure 2 we report the average position $\langle h(x, t)\rangle_{F_{0}}$ for different $x$ : the agreement with the theoretical estimation given in (76) and (77) is excellent.

We now calculate the complex mobility according to (58). Plugging in the values of the parameters we have

$$
\mu\left(x, \omega_{0}\right)=\frac{1}{2} \sqrt{\frac{-i \omega_{0}}{\xi}} e^{-\left|x-x^{\star}\right| \sqrt{-i \omega_{0} \xi}}
$$

which can be recasted as

$$
\mu\left(x, \omega_{0}\right)=\frac{1}{2} \sqrt{\frac{\omega_{0}}{\xi}} e^{-\left|x-x^{\star}\right| \sqrt{\omega_{0} \xi / 2}} e^{i\left(\left|x-x^{\star}\right| \sqrt{\omega_{0} \xi / 2}-\pi / 4\right)} .
$$




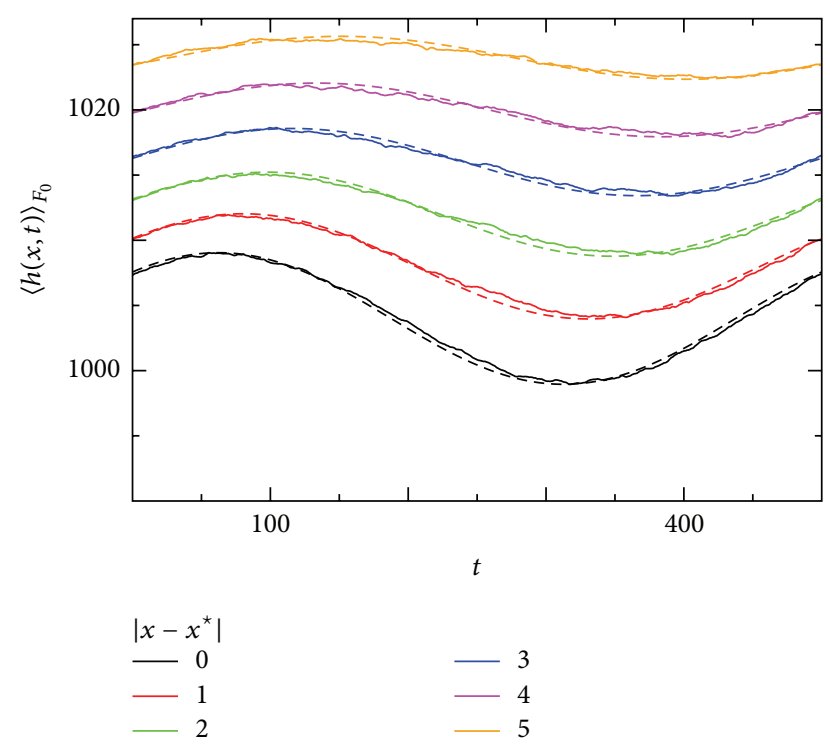

Figure 2: (Color online) Average particle drift $\langle h(x, t)\rangle_{F_{0}}$ for an applied time periodic force in single file systems. The simulations were carried out in the following way: a file of $N=501$ Brownian nontrespassing particles was moving along a ring of length $L=$ $5010(\rho=N / L=0.1)$. To the middle particle $\left(x^{\star}=251\right) \mathrm{a}$ periodic driving force $F_{0} \cos \left(\omega_{0} t\right)$ was applied with $F_{0}=0.2$ and $\omega_{0}=0.012566368$. In the figure the average positions of the particles whose distance from $x^{\star}\left(\left|x-x^{\star}\right|\right)$ ranges from 1 to 5 are shown: the black solid curve represents the average drift $\left\langle h\left(x^{\star}, t\right)\right\rangle_{F_{0}}$, while the dashed lines stand for the theoretical predictions equations (76) and (77), respectively. Statistical averages were taken over 50 different system's realizations and then averaged over more than 2000 periods $T=(2 \pi) / \omega_{0}=500$. The other simulation parameters are $\xi=0.5$ and $k_{B} T=10$.

Furthermore for the mobility of the $x^{\star}$ th particle we achieve

$$
\mu\left(x^{\star}, \omega_{0}\right)=\frac{1}{2} \sqrt{\frac{\omega_{0}}{\xi}} e^{-i \pi / 4} ;
$$

this result has been firstly obtained and numerically proven in [38] and has been rigorously derived in [39].

\section{Conclusions}

In this paper we have studied the scaling properties of the average velocity drifts for a tagged and untagged probe particle in a generalized elastic model, where a time periodic localized force is supposed to operate. The tagged probe is considered to be the point on which the external force acts, while the untagged probe is any other point on the system, which is secondarily affected by the action of the perturbation. Within the FLE framework, the stochastic motion of both tracers evidently appears to be influenced by the external force, since the external perturbation propagates through the system and the surrounding medium. This propagation is mathematically expressed by the noiseand force-propagators (Green's functions), carrying the perturbation between two points of the system, in a certain lapse of time. We analyzed the double scaling behaviour of these propagators which arise naturally thanks to Fox $H$ function formalism. We have shown that such behaviour affects the stochastic dynamics of both tracers. In particular, we demonstrated how the response of the untagged tracer differs drastically in the high or low frequency limits of the applied perturbation, when hydrodynamic interactions can be considered long ranged or local. In particular, we have shown that the validity of the Kubo fluctuation relations and of the Green-Kubo relations leads to the emergence of a physical picture far from being trivial. As a matter of fact, we have shown that the physical system splits into two regions, where the amplitude and the phase shift of the response signal can be very different according to the parameters characterizing the GEM dynamics. Moreover the size of these regions can be varied straightforwardly by tuning the frequency of the perturbation applied.

\section{Appendices}

\section{A. Fox Function Properties}

In this section we enumerate the properties of the Fox functions that we use throughout our analysis. This list is not an exhaustive compendium of the Fox functions properties, for which the reader could refer to $[36,37,52]$. Some useful properties are also reported in [24].

For convenience in this section we adopt the following short notation:

$$
\begin{gathered}
H_{p q}^{m n}\left[y \mid \begin{array}{ccc}
\left(a_{1}, A_{1}\right) & \cdots & \left(a_{p}, A_{p}\right) \\
\left(b_{1}, B_{1}\right) & \cdots & \left(b_{q}, B_{q}\right)
\end{array}\right] \\
=H_{p q}^{m n}\left[\begin{array}{c}
\left.y \mid \begin{array}{c}
\left.a_{p}, A_{p}\right] \\
{\left[b_{q}, B_{q}\right]}
\end{array}\right] .
\end{array}\right.
\end{gathered}
$$

The useful rules are hereafter listed:

$$
\begin{aligned}
& H_{p q}^{m n}\left[y \mid \begin{array}{c}
{\left[a_{p}, A_{p}\right]} \\
{\left[b_{q}, B_{q}\right]}
\end{array}\right]=H_{q p}^{n m}\left[\begin{array}{c}
1 \\
y
\end{array} \mid \begin{array}{c}
{\left[1-b_{q}, B_{q}\right]} \\
{\left[1-a_{p}, A_{p}\right]}
\end{array}\right], \\
& H_{p q}^{m n}\left[y \mid \begin{array}{c}
{\left[a_{p}, A_{p}\right]} \\
{\left[b_{q}, B_{q}\right]\left(a_{1}, A_{1}\right)}
\end{array}\right]=H_{p-1 q-1}^{m n-1}\left[\begin{array}{c}
y \\
\left(a_{2}, A_{2}\right), \ldots,\left(a_{p}, A_{p}\right) \\
{\left[b_{q-1}, B_{q-1}\right]}
\end{array}\right],
\end{aligned}
$$




$$
\begin{aligned}
& \int_{0}^{\infty} y^{\alpha-1} \cos (\sigma y) H_{p q}^{m n}\left[\omega y^{r} \mid \begin{array}{c}
{\left[a_{p}, A_{p}\right]} \\
{\left[b_{q}, B_{q}\right]}
\end{array}\right] d y
\end{aligned}
$$

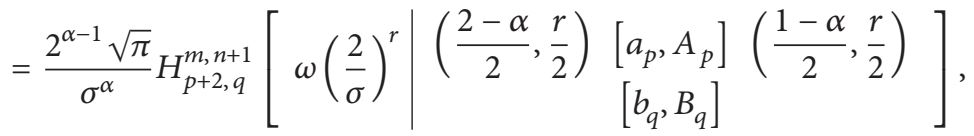

$$
\begin{aligned}
& \frac{1}{k} H_{p q}^{m n}\left[y \mid \begin{array}{c}
{\left[a_{p}, A_{p}\right]} \\
{\left[b_{q}, B_{q}\right]}
\end{array}\right]=H_{p q}^{m n}\left[\begin{array}{c|c}
y^{k} & {\left[a_{p}, k A_{p}\right]} \\
{\left[b_{q}, k B_{q}\right]}
\end{array}\right], \\
& y^{\sigma} H_{p q}^{m n}\left[\begin{array}{c|c}
{\left[a_{p}, A_{p}\right]} \\
{\left[b_{q}, B_{q}\right]}
\end{array}\right]=H_{p q}^{m n}\left[\begin{array}{c}
y \\
{\left[a_{p}+\sigma A_{p}, A_{p}\right]} \\
{\left[b_{q}+\sigma B_{q}, B_{q}\right]}
\end{array}\right] \text {, } \\
& \int_{0}^{\infty} y^{\alpha-1} J_{v}(\sigma y) H_{p q}^{m n}\left[\omega y^{r} \mid \begin{array}{c}
{\left[a_{p}, A_{p}\right]} \\
{\left[b_{q}, B_{q}\right]}
\end{array}\right] d y \\
& =\frac{2^{\alpha-1}}{\sigma^{\alpha}} H_{p+2, q}^{m, n+1}\left[\omega\left(\frac{2}{\sigma}\right)^{r} \mid\left(1-\frac{\alpha+v}{2}, \frac{r}{2}\right) \underset{\left[a_{p}, A_{p}\right]}{\left[b_{q}, B_{q}\right]}\left(1-\frac{\alpha-v}{2}, \frac{r}{2}\right)\right] \text {, } \\
& { }_{0} D_{t}^{v} y^{\alpha} H_{p q}^{m n}\left[(\sigma y)^{\beta} \mid \begin{array}{c}
{\left[a_{p}, A_{p}\right]} \\
{\left[b_{q}, B_{q}\right]}
\end{array}\right]=\omega^{\alpha-v} H_{p+1}^{m+n+1}\left[\begin{array}{c|c|c|}
(\sigma y)^{\beta} & (-\alpha, \beta) & {\left[a_{p}, A_{p}\right]} \\
{\left[b_{q}, B_{q}\right]} & (\nu-\alpha, \beta)
\end{array}\right] \text {, } \\
& H_{01}^{10}\left[\begin{array}{l|c}
- & - \\
{\left[b_{q}, B_{q}\right]}
\end{array}\right]=\frac{y^{b / B}}{B} e^{-y^{1 / B}} \text {. }
\end{aligned}
$$

Asymptotic expansion is $y \rightarrow 0$,

$$
\begin{aligned}
H_{p q}^{m n}\left[y \mid \begin{array}{c}
{\left[a_{p}, A_{p}\right]} \\
{\left[b_{q}, B_{q}\right]}
\end{array}\right] & =\sum_{i=1}^{m} \sum_{k=0}^{\infty} c_{i k} \frac{(-1)^{k}}{k ! B_{i}} y^{\left(b_{i}+k\right) / B_{i}}, \\
c_{i k} & =\frac{\prod_{j=1, j \neq i}^{m} \Gamma\left(b_{j}-\left(b_{i}+k\right) B_{j} / B_{i}\right) \prod_{j=1}^{n} \Gamma\left(1-a_{j}+\left(b_{i}+k\right) A_{j} / B_{i}\right)}{\prod_{j=m+1}^{q} \Gamma\left(1-b_{j}+\left(b_{i}+k\right) B_{j} / B_{i}\right) \prod_{j=n+1}^{p} \Gamma\left(a_{j}-\left(b_{i}+k\right) A_{j} / B_{i}\right)} .
\end{aligned}
$$

This expansion is valid whenever $\sum_{i=1}^{q} B_{i}-\sum_{i=1}^{p} A_{i} \geq 0$ or $\sum_{i=1}^{q} B_{i}-\sum_{i=1}^{p} A_{i}<0$ and $\sum_{i=1}^{n} A_{i}-\sum_{i=n+1}^{p} A_{i}+\operatorname{sum}_{i=1}^{m} B_{i}-$ Asymptotic expansion is $y \rightarrow \infty$, $\operatorname{sum}_{i=m+1}^{q} B_{i}>0$.

$$
\begin{aligned}
H_{p q}^{m n}\left[y \mid \begin{array}{c}
{\left[a_{p}, A_{p}\right]} \\
{\left[b_{q}, B_{q}\right]}
\end{array}\right] & =\sum_{i=1}^{n} \sum_{k=0}^{\infty} c_{i k} \frac{(-1)^{k}}{k ! A_{i}} y^{-\left(1-a_{i}+k\right) / A_{i}}, \\
c_{i k} & =\frac{\prod_{j=1, j \neq i}^{n} \Gamma\left(1-a_{j}-\left(1-a_{i}+k\right) A_{j} / A_{i}\right) \prod_{j=1}^{m} \Gamma\left(b_{j}+\left(1-a_{i}+k\right) B_{j} / A_{i}\right)}{\prod_{j=n+1}^{q} \Gamma\left(a_{j}+\left(1-a_{i}+k\right) A_{j} / A_{i}\right) \prod_{j=m+1}^{q} \Gamma\left(1-b_{j}-\left(1-a_{i}+k\right) B_{j} / A_{i}\right)} .
\end{aligned}
$$

This expansion is valid whenever $\sum_{i=1}^{q} B_{i}-\sum_{i=1}^{p} A_{i} \leq 0$ or $\sum_{i=1}^{q} B_{i}-\sum_{i=1}^{p} A_{i}>0$ and $\sum_{i=1}^{n} A_{i}-\sum_{i=n+1}^{p} A_{i}+\operatorname{sum}_{i=1}^{m} B_{i}-$ $\operatorname{sum}_{i=m+1}^{q} B_{i}>0$. Empty products are interpreted as being unity. 


\section{B. Asymptotic Solution of Fourier Integrals}

The integrals appearing throughout the paper are of two types. The first is

$$
I(\lambda)=\int_{0}^{+\infty} y^{d / 2} J_{(d / 2)-1}(\lambda y) e^{-y^{z+\alpha-d}} d y,
$$

with $\lambda=(\tau / t)^{2 / \gamma}$. We furnish the solution of (B.1) in the limit of $\lambda \rightarrow \infty$. The integral can be evaluated by expanding the exponential for small arguments; that is, $e^{-y^{z+\alpha-d}} \simeq 1-y^{z+\alpha-d}$. The first term gives zero contribution, while the second is [53]

$$
\begin{aligned}
& I(\lambda) \simeq-\int_{0}^{+\infty} y^{z+\alpha-d / 2} J_{d / 2-1}(\lambda y) d y=\frac{2^{z+\alpha-d / 2-2} \gamma}{\pi} \\
& \cdot \sin \left(\frac{\pi \gamma}{4}\right) \Gamma\left(\frac{z+\alpha}{2}\right) \Gamma\left(\frac{\gamma}{4}\right) \lambda^{1+(d+2) / 4} .
\end{aligned}
$$

The second integral has the following form:

$$
I(\lambda)=\int_{0}^{+\infty} y^{\alpha-d / 2} J_{d / 2-1}(\lambda y) e^{-y^{z+\alpha-d}} d y .
$$

Expanding the exponential for small arguments and retaining the first term, we obtain [53]

$$
\begin{aligned}
I(\lambda) & \simeq \int_{0}^{+\infty} y^{\alpha-d / 2} J_{d / 2-1}(\lambda y) d y \\
& =2^{\alpha-d / 2} \frac{\Gamma(\alpha / 2)}{\Gamma((d-\alpha) / 2)} \lambda^{(2 \alpha-d+2) / \gamma}
\end{aligned}
$$

\section{Laplace's Method for Asymptotic Integrals}

In this appendix we report the theorem for the asymptotic solution of exponential integrals through Laplace method [54].

Consider the integral

$$
I(t)=\int_{a}^{b} x(q) e^{-p(q) t} d q .
$$

If the hypotheses

(i) $p(q)>p(a)$ for any $q \in(a, b)$ and the minimum of $p(q)$ is approached only at $a$,

(ii) $d p(q) / d q$ and $x(q)$ are continuous functions in a neighborhood of $a$, except, possibly, at $a$,

(iii) as $q \rightarrow a^{-},(p(q)-p(a)) \sim P(q-a)^{\mu}$ and $x(q) \sim \sim$ $Q(q-a)^{\lambda-1}$, where $P, \mu$, and $\lambda$ are positive constant and $Q \in \mathbb{R}$ or $\in \mathbb{C}$,

(iv) $I(t)$ is absolutely convergent throughout its range for all sufficiently large $t$

are fulfilled, then the integral $I(t)$ is

$$
I(t) \simeq \frac{Q}{\mu} \Gamma\left(\frac{\lambda}{\mu}\right) \frac{e^{-p(a) t}}{(P t)^{\lambda / \mu}}
$$

in the limit $t \rightarrow \infty$.

\section{Asymptotic Form of the Fractional Derivative of an Exponential}

We want to calculate the following expression:

$$
{ }_{0} D_{t}^{\beta} e^{-a t}=\frac{1}{\Gamma(1-\beta)} \frac{d}{d t} \int_{a}^{t} \frac{e^{-a t^{\prime}}}{\left(t-t^{\prime}\right)^{\beta}} d t^{\prime} .
$$

By solving explicitly the integral [37] we obtain

$$
\begin{aligned}
{ }_{0} D_{t}^{\beta} e^{-a t} \\
\quad=\frac{1}{\Gamma(1-\beta)} \frac{d}{d t}\left[(-a)^{\beta-1} e^{-a t} \gamma(1-\beta,-a t)\right],
\end{aligned}
$$

where $\gamma$ represents the incomplete gamma function [53]. Performing the time derivative we cast the result in the following form:

$$
\begin{aligned}
& { }_{0} D_{t}^{\beta} e^{-a t} \\
& \quad=\frac{1}{\Gamma(1-\beta)}\left[(-a)^{\beta} e^{-a t} \gamma(1-\beta,-a t)+t^{-\beta}\right],
\end{aligned}
$$

and, by using the asymptotic expansion of the incomplete gamma function [53], we finally get

$$
{ }_{0} D_{t}^{\beta} e^{-a t} \simeq(-a)^{\beta} e^{-a t}+\frac{t^{-\beta-1}}{a \Gamma(-\beta)} .
$$

\section{Conflict of Interests}

The author declares that there is no conflict of interests regarding the publication of this paper.

\section{Acknowledgments}

The author acknowledges the financial support of the ERC advanced Grant SIZEFFECTS. The author is also deeply indebted to Professor A. Chechkn for having substantially contributed to the elaboration of the present analysis.

\section{References}

[1] A. Taloni, A. Chechkin, and J. Klafter, "Generalized elastic model yields a fractional langevin equation description," Physical Review Letters, vol. 104, no. 16, Article ID 160602, 2010.

[2] A. I. Saichev and G. M. Zaslavsky, "Fractional kinetic equations: solutions and applications," Chaos, vol. 7, no. 4, pp. 753-764, 1997.

[3] S. G. Samko, A. A. Kilbas, and O. I. Marichev, Fractional Integrals and Derivatives, Theory and Applications, Gordon and Breach Science Publishers, Yverdon-les-Bains, Switzerland, 1993.

[4] M. Doi and S. F. Edwards, The Theory of Polymer Dynamics, vol. 73, Oxford University Press, 1988.

[5] R. Granek, "From semi-flexible polymers to membranes: anomalous diffusion and reptation," Journal de Physique II, vol. 7, no. 12, pp. 1761-1788, 1997. 
[6] E. Farge and A. C. Maggs, "Dynamic scattering from semiflexible polymers," Macromolecules, vol. 26, no. 19, pp. 5041-5044, 1993.

[7] A. Caspi, M. Elbaum, R. Granek, A. Lachish, and D. Zbaida, "Semiflexible polymer network: a view from inside," Physical Review Letters, vol. 80, no. 5, pp. 1106-1109, 1998.

[8] S. F. Edwards and D. Wilkinson, "The surface statistics of a granular aggregate," Proceedings of the Royal Society of London A: Mathematical, Physical and Engineering Sciences, vol. 381, no. 1780, pp. 17-31, 1982.

[9] P. E. Rouse Jr., "A theory of the linear viscoelastic properties of dilute solutions of coiling polymers," The Journal of Chemical Physics, vol. 21, no. 7, pp. 1272-1280, 1953.

[10] B. H. Zimm, "Dynamics of polymer molecules in dilute solution: viscoelasticity, flow birefringence and dielectric loss," The Journal of Chemical Physics, vol. 24, no. 2, pp. 269-278, 1956.

[11] É. Freyssingeas, D. Roux, and F. Nallet, "Quasi-elastic light scattering study of highly swollen lamellar and 'sponge' phases," Journal de Physique II, vol. 7, no. 6, pp. 913-929, 1997.

[12] E. Helfer, S. Harlepp, L. Bourdieu, J. Robert, F. C. MacKintosh, and D. Chatenay, "Microrheology of biopolymer-membrane complexes," Physical Review Letters, vol. 85, no. 2, pp. 457-460, 2000.

[13] R. Granek and J. Klafter, "Anomalous motion of membranes under a localized external potential," Europhysics Letters, vol. 56, no. 1, pp. 15-21, 2001.

[14] A. G. Zilman and R. Granek, "Membrane dynamics and structure factor," Chemical Physics, vol. 284, no. 1-2, pp. 195-204, 2002.

[15] A. G. Zilman and R. Granek, "Dynamics of fractal sol-gel polymeric clusters," Physical Review E, vol. 58, no. 3, Article ID R2725, 1998.

[16] P. C. Searson, R. Li, and K. Sieradzki, "Surface diffusion in the solid-on-solid model," Physical Review Letters, vol. 74, no. 8, pp. 1395-1398, 1995.

[17] J. Krug and H. T. Dobbs, "Anomalous tracer diffusion on surfaces," Physical Review Letters, vol. 76, no. 21, article 4096, 1996.

[18] S. N. Majumdar and A. J. Bray, "Spatial persistence of fluctuating interfaces," Physical Review Letters, vol. 86, no. 17, pp. 37003703, 2001.

[19] J. Krug, "Origins of scale invariance in growth processes," Advances in Physics, vol. 46, no. 2, pp. 139-282, 1997.

[20] Z. Toroczkai and E. D. Williams, "Nanoscale fluctuations at solid surfaces," Physics Today, vol. 52, no. 12, pp. 24-28, 1999.

[21] S. Majaniemi, T. Ala-Nissila, and J. Krug, "Kinetic roughening of surfaces: derivation, solution, and application of linear growth equations," Physical Review B, vol. 53, no. 12, pp. 80718082, 1996.

[22] H. Gao and J. R. Rice, "First-order perturbation analysis of crack trapping by arrays of obstacles," Journal of Applied MechanicsTransactions ASME, vol. 56, no. 4, pp. 828-836, 1989.

[23] J. F. Joanny and P. G. De Gennes, "A model for contact angle hysteresis," The Journal of Chemical Physics, vol. 81, no. 1, pp. 552-562, 1984.

[24] A. Taloni, A. Chechkin, and J. Klafter, "Unusual response to a localized perturbation in a generalized elastic model," Physical Review E, vol. 84, no. 2, Article ID 021101, 2011.

[25] A. Taloni, A. Chechkin, and J. Klafter, "Generalized elastic model: fractional Langevin description, fluctuation relation and linear response," Mathematical Modelling of Natural Phenomena, vol. 8, no. 2, pp. 127-143, 2013.

[26] W. G. Glöckle and T. F. Nonnenmacher, "Fractional integral operators and fox functions in the theory of viscoelasticity," Macromolecules, vol. 24, no. 24, pp. 6426-6434, 1991.

[27] W. G. Glöckle and T. F. Nonnenmacher, "Fox function representation of non-debye relaxation processes," Journal of Statistical Physics, vol. 71, no. 3-4, pp. 741-757, 1993.

[28] R. Metzler, W. G. Glöckle, and T. F. Nonnenmacher, "Fractional model equation for anomalous diffusion," Physica A: Statistical Mechanics and its Applications, vol. 211, no. 1, pp. 13-24, 1994.

[29] R. Metzler and J. Klafter, "The random walk's guide to anomalous diffusion: a fractional dynamics approach," Physics Reports, vol. 339, no. 1, pp. 1-77, 2000.

[30] F. Mainardi, G. Pagnini, and R. Saxena, "Fox H functions in fractional diffusion," Journal of Computational and Applied Mathematics, vol. 178, no. 1-2, pp. 321-331, 2005.

[31] S. I. Denisov, S. B. Yuste, Y. S. Bystrik, H. Kantz, and K. Lindenberg, "Asymptotic solutions of decoupled continuoustime random walks with superheavy-tailed waiting time and heavy-tailed jump length distributions," Physical Review E, vol. 84, no. 6, Article ID 061143, 2011.

[32] R. K. Saxena, A. M. Mathai, and H. J. Haubold, "Fractional reaction-diffusion equations," Astrophysics and Space Science, vol. 305, no. 3, pp. 289-296, 2006.

[33] M. O. Vlad, R. Metzler, and J. Ross, "Generalized Huber kinetics for nonlinear rate processes in disordered systems: nonlinear analogs of stretched exponential," Physical Review E, vol. 57, no. 6, pp. 6497-6505, 1998.

[34] N. Laskin, "Fractional quantum mechanics and Lévy path integrals," Physics Letters A, vol. 268, no. 4-6, pp. 298-305, 2000.

[35] A. A. Kilbas, H-Transforms: Theory and Applications, CRC Press, New York, NY, USA, 2004.

[36] A. M. Mathai, R. K. Saxena, and H. J. Haubold, The H-Function: Theory and Applications, Springer, 2009.

[37] A. Prudnikov, Integrals and Series: Special Functions, CRC Press, New York, NY, USA, 1998.

[38] A. Taloni and M. A. Lomholt, "Langevin formulation for singlefile diffusion," Physical Review E, vol. 78, no. 5, Article ID 051116, 2008.

[39] L. Lizana, T. Ambjörnsson, A. Taloni, E. Barkai, and M. A. Lomholt, "Foundation of fractional Langevin equation: harmonization of a many-body problem," Physical Review E, vol. 81, no. 5, Article ID 051118, 2010.

[40] D. Panja, "Generalized langevin equation formulation for anomalous polymer dynamics," Journal of Statistical Mechanics: Theory and Experiment, vol. 2010, no. 2, Article ID L02001, 2010.

[41] I. Podlubny, Fractional Differential Equations: An Introduction to Fractional Derivatives, Fractional Differential Equations, to Methods of Their Solution and Some of Their Applications, vol. 198, Academic Press, New York, NY, USA, 1998.

[42] M. Caputo, "Linear models of dissipation whose $Q$ is almost frequency independent-II," Geophysical Journal International, vol. 13 , no. 5, pp. 529-539, 1967.

[43] A. Taloni, A. Chechkin, and J. Klafter, "Generalized elastic model: thermal vs. non-thermal initial conditions-universal scaling, roughening, ageing and ergodicity," Europhysics Letters, vol. 97, no. 3, Article ID 30001, 2012.

[44] A. Taloni, A. Chechkin, and J. Klafter, "Correlations in a generalized elastic model: fractional Langevin equation approach," Physical Review E, vol. 82, no. 6, Article ID 061104, 2010. 
[45] F. Family and T. Vicsek, "Scaling of the active zone in the Eden process on percolation networks and the ballistic deposition model," Journal of Physics A: Mathematical and General, vol. 18, no. 2, pp. L75-L81, 1985.

[46] J. M. López, M. A. Rodríguez, and R. Cuerno, "Power spectrum scaling in anomalous kinetic roughening of surfaces," Physica A: Statistical Mechanics and Its Applications, vol. 246, no. 3-4, pp. 329-347, 1997.

[47] J. J. Ramasco, J. M. López, and M. A. Rodríguez, "Generic dynamic scaling in kinetic roughening," Physical Review Letters, vol. 84, no. 10, pp. 2199-2202, 2000.

[48] R. Kubo, M. Toda, and N. Hashitsume, Statistical Physics II: Nonequilibrium Statistical Mechanics, vol. 31, Springer Science \& Business Media, 2012.

[49] U. M. B. Marconi, A. Puglisi, L. Rondoni, and A. Vulpiani, "Fluctuation-dissipation: response theory in statistical physics," Physics Reports, vol. 461, no. 4-6, pp. 111-195, 2008.

[50] D. Villamaina, A. Baldassarri, A. Puglisi, and A. Vulpiani, "The fluctuation-dissipation relation: how does one compare correlation functions and responses?" Journal of Statistical Mechanics: Theory and Experiment, vol. 2009, no. 7, Article ID P07024, 2009.

[51] I. S. Gradshtein and I. M. Ryzhik, Tables of Integrals, Sums, Series, and Products, Fizmatgiz, Moscow, Russia, 1971.

[52] R. Hilfer, P. Butzer, U. Westphal et al., Applications of Fractional Calculus in Physics, World Scientific, River Edge, NJ, USA, 2000.

[53] M. Abramowitz and I. A. Stegun, Handbook of Mathematical Functions: with Formulas, Graphs, and Mathematical Tables, vol. 55, Courier Corporation, 1964.

[54] F. W. Olver, Asymptotics and Special Functions, Academic Press, New York, NY, USA, 2014. 


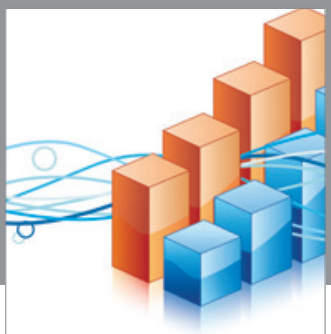

Advances in

Operations Research

vatem alat4

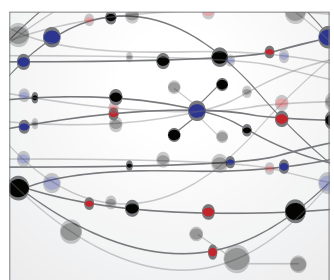

\section{The Scientific} World Journal
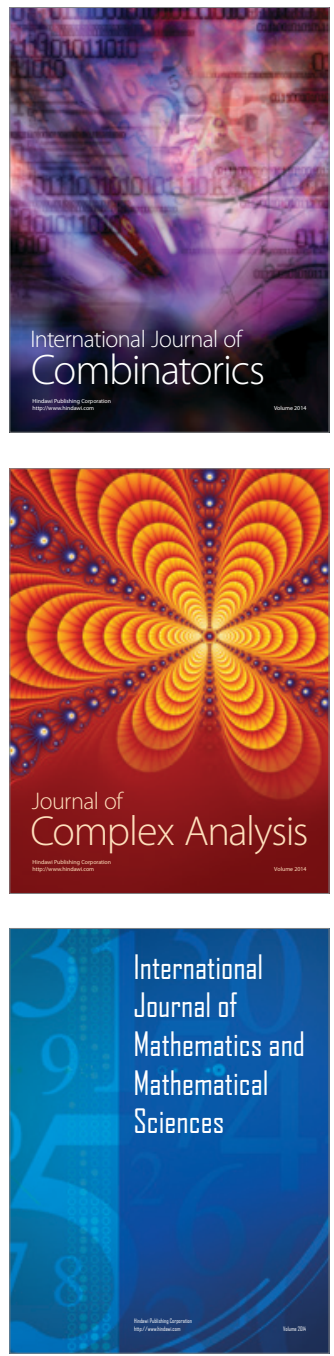
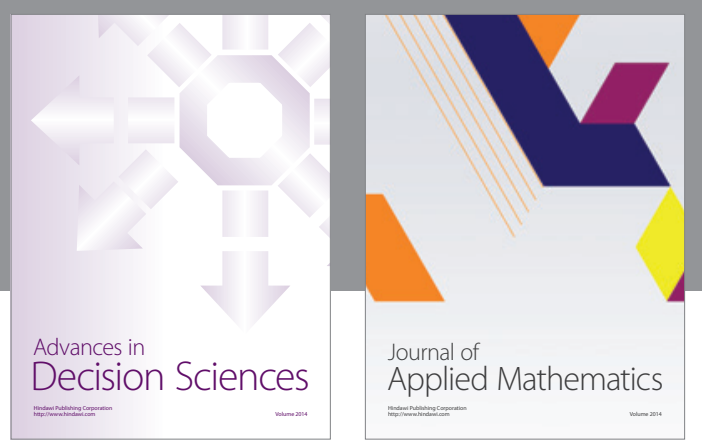

Algebra

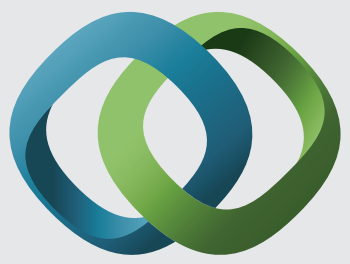

\section{Hindawi}

Submit your manuscripts at

http://www.hindawi.com
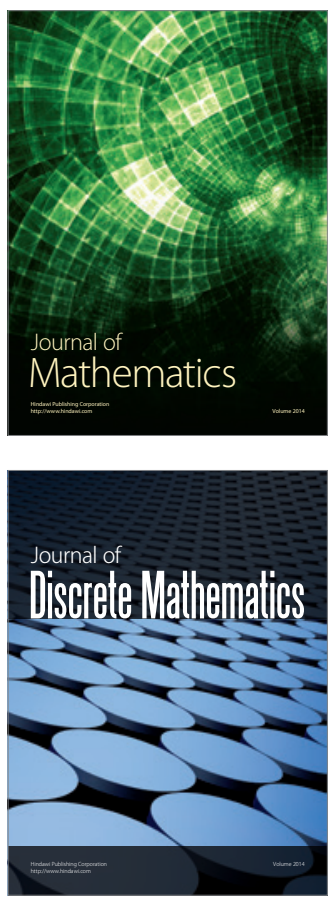

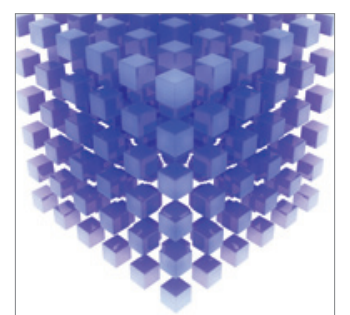

Mathematical Problems in Engineering
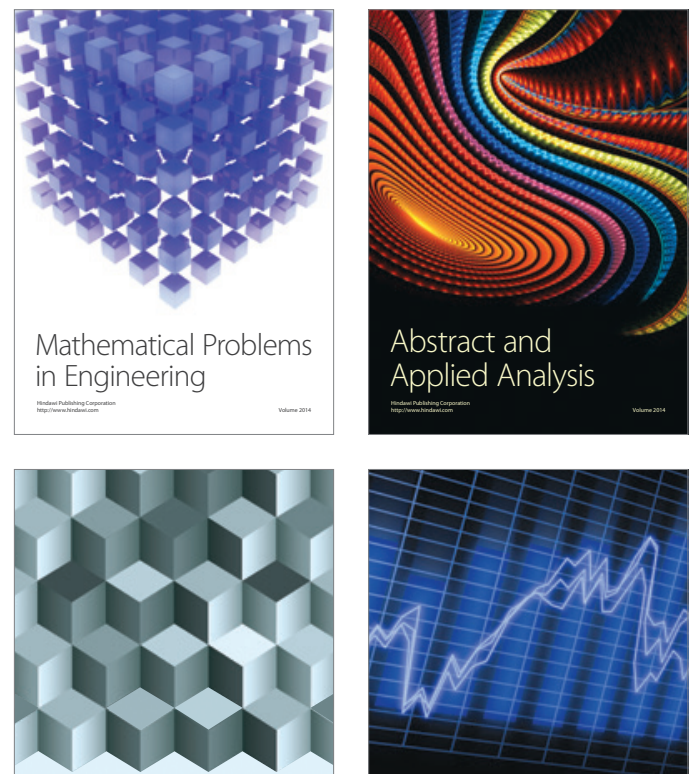

Journal of

Function Spaces

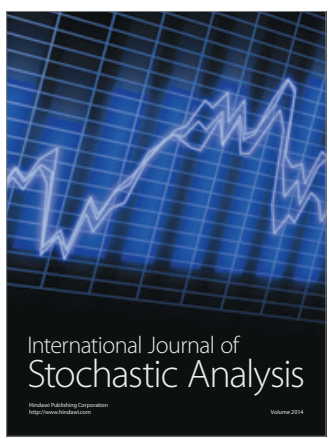

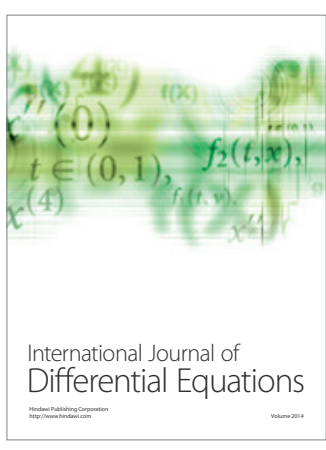
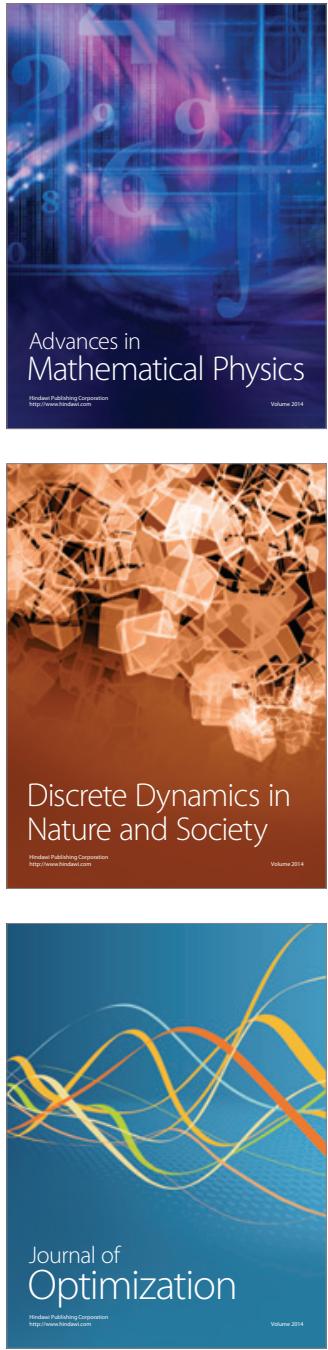